\title{
An Anti-Inflammatory Sterol Decreases Obesity-Related Inflammation-Induced Insulin Resistance and Metabolic Dysregulation
}

\author{
Chris L. Reading, Jaime Flores-Riveros, Dwight R. Stickney, and James M. Frincke \\ Harbor Therapeutics, Inc., San Diego, CA 92122, USA \\ Correspondence should be addressed to Chris L. Reading; creading@harbortx.com
}

Received 16 November 2012; Revised 18 December 2012; Accepted 20 December 2012

Academic Editor: François Mach

Copyright (C) 2013 Chris L. Reading et al. This is an open access article distributed under the Creative Commons Attribution License, which permits unrestricted use, distribution, and reproduction in any medium, provided the original work is properly cited.

Obesity-related inflammation-induced insulin resistance and metabolic dysregulation were investigated in retrospective analysis of placebo hematologic and metabolic laboratory data from trials associated with increasing chronic low-grade inflammation and body mass index. Studies included healthy subjects and those with progressive stages of metabolic dysregulation, including type 2 diabetes mellitus with uncontrolled hemoglobin $A 1_{c}$. Intrasubject variances in erythroid and metabolic values increased with metabolic dysregulation. Random effects were demonstrated in treatment-naïve diabetes for erythroid, glucose, and HbA1c fluctuations. The anti-inflammatory insulin sensitizer, HE3286, was tested for its ability to decrease obesity-related inflammationinduced insulin resistance and metabolic dysregulation in diabetes. HE3286 significantly decreased erythroid and metabolic variances and improved 1,5-anhydroglucitol (a surrogate of postprandial glucose) compared to the placebo group. HE3286 $\mathrm{HbA1c}$ decrease correlated with weight loss and inversely with baseline monocyte chemoattractant protein-1 (MCP-1) in metformintreated diabetics. Normalization of HbA1c to the 84-day average hemoglobin revealed that HE3286 HbA1c decrease correlated with high baseline MCP-1 and MCP-1 decrease in treatment-naïve diabetics. HE3286 decreased insulin resistance, increased the frequency of decreased day $84 \mathrm{HbA1c}$ in metformin-treated subjects, and decreased day $112 \mathrm{HbA1c}$ in treatment-naïve diabetics. HE3286 may be useful to restore metabolic homeostasis in type 2 diabetes.

\section{Introduction and Purpose}

HE3286 (17 $\alpha$-ethynylandrost-5-ene- $3 \beta, 7 \beta, 17 \beta$-triol) is a chemical derivative of the natural mammalian sterol androst-5-ene-3 $\beta, 7 \beta, 17 \beta$-triol ( $\beta$ AET). $\beta$ AET exhibits antiinflammatory activity in rodent models, is elevated in plasma of obese subjects with normal glucose disposal, and may play a compensatory role in preventing development of metabolic syndrome (reviewed in [1]). $\beta$ AET is pharmaceutically unsuitable, due to poor oral bioavailability and its propensity for oxidative inactivation by $17 \beta$-hydroxysteroid dehydrogenase [1]. HE3286 is stabilized against oxidation at position 17 and consequently orally bioavailable, does not bind to any known nuclear steroid hormone receptors, and is pharmacologically unrelated to androgens, estrogens, corticosteroids, or peroxisome proliferators [1]. HE3286 has shown broad anti-inflammatory activity in animal models of rheumatoid arthritis, ulcerative colitis, multiple sclerosis, lung inflammation, autoimmune type 1 diabetes, and neuroinflammation (reviewed in [1]). In these models, nuclear factor kappa $\mathrm{B}(\mathrm{NF} \kappa \mathrm{B})$ activation and proinflammatory cytokine production were consistently suppressed. Furthermore, HE3286 was not markedly immunosuppressive in rodent models of ovalbumin immunization, Klebsiella pneumoniae or Pseudomonas aeruginosa infection, Coxsackievirus B3 myocarditis, delayed-type hypersensitivity, and mitogeninduced proliferation, or in the human mixed lymphocyte reaction assay (reviewed in [1]).

Obesity induces an insulin-resistant state in adipose tissue [2], liver, and muscle and is a strong risk factor for the development of type 2 diabetes mellitus [3]. In adipose tissue, MCP-1 and tumor necrosis factor alpha (TNF $\alpha$ ) play 
dominant proinflammatory roles [2]. Adiposity-induced inflammation-stimulated kinases phosphorylate insulin receptor substrate-1 on serine residues and inhibit insulin signaling [4]. Two recent publications report the activity of HE3286 against in vitro inflammatory responses and in vivo rodent models of obesity-induced inflammation and insulin resistance $[5,6]$. HE3286 suppressed endotoxininduced $\mathrm{NF} \kappa \mathrm{B}$ activation, reporter gene expression, nuclear localization, and p65 phosphorylation in mouse macrophages and decreased phosphorylation of the proinflammatory extracellular signal-regulated (Erk1/2), IkappaB (Ikk), Jun N-terminal (Jnk), and p38 mitogenactivated protein (p38 Mapk) kinases. HE3286 also attenuated $\mathrm{TNF} \alpha$-stimulated inflammation and $\mathrm{TNF} \alpha$ induced adipocyte-stimulated macrophage chemotaxis $[5,6]$. HE3286 treatment of diabetic $d b / d b$ mice, insulinresistant diet-induced obese mice, and genetically obese $o b / o b$ mice suppressed progression to hyperglycemia and markedly improved glucose clearance. This effect appeared to be consequent to reduced insulin resistance, since HE3286 lowered blood insulin levels in both $d b / d b$ and $o b / o b$ mice. In these studies HE3286 suppressed levels of the chemokine monocyte chemoattractant protein-1 (MCP-1), along with its cognate receptor, $\mathrm{C}-\mathrm{C}$ motif chemokine receptor-2, in white adipose tissue [6]. In Zucker diabetic fatty rats, HE3286 downregulated inflammatory cytokine and chemokine expression in both liver and adipose tissues and suppressed macrophage migration into adipose tissue. HE3286 normalized fasting and fed glucose levels, improved glucose tolerance, and enhanced skeletal muscle and liver insulin sensitivity, as assessed by hyperinsulinemic, euglycemic clamp studies. In addition, HE3286 reduced liver cholesterol and triglyceride content, leading to a feedback elevation of low-density lipoprotein (LDL) receptor and decreased total serum cholesterol [5]. Recently, we have reported that HE3286 binds to Erk1/2, Lrp1, and Sirt2 [7] and proposed that the HE3286-mediated decrease in hyperactivation of Erk1/2 may be causal for its metabolic [8] and anti-inflammatory activities.

In a clinical study in obese, impaired glucose tolerance (IGT) subjects, HE3286 significantly increased the frequency of insulin-resistant subjects with improved day 29 insulinstimulated glucose disposal, increased HDL cholesterol, and decreased day 28 CRP compared to placebo-treated subjects [9]. Based on baseline glucose clamp studies, insulin-resistant subjects had elevated inflammatory biomarkers, with lower adiponectin and higher cytokine secretion in LPS-stimulated PBMC. After 28 days of HE3286 treatment, adiponectin levels increased significantly in insulin-resistant subjects, compared to placebo. These results support our hypothesis that obesity-induced inflammation is a significant contributor to metabolic dysregulation and that the anti-inflammatory activity of HE3286 can preferentially benefit the insulinresistant inflamed subpopulation of obese IGT subjects.

Based on preclinical studies and these foregoing results in IGT subjects, it was conjected that HE3286 might benefit obese inflamed insulin-resistant individuals with type 2 diabetes mellitus (T2DM). A widely accepted clinical endpoint for $\mathrm{T} 2 \mathrm{DM}$ is the change in $\mathrm{HbA1c}$, a surrogate marker for the extent of hyperglycemia an individual experiences over time. Traditionally, erythroid hematology values are considered stable in healthy individuals, and hemoglobin and $\mathrm{HbA} 1 \mathrm{c}$ turnover is reported to reflect the normal red cell half-life of 38-60 days [10]. In T2DM, the life span of red cells can be altered significantly by inflammation, particularly TNF $\alpha$-induced oxidative stress [11], obese lowgrade systemic inflammatory response syndrome [12], the presence of elevated levels of advanced glycation endproducts on the surface of red cells $[13,14]$, hypoxia [15], and excessive erythrocytosis [16]. There are reports of large fluctuations in HbA1c in type 1 diabetes [17], especially in subjects with poor glycemic control $[18,19]$. This information prompted us to also assess the association of obesity-related chronic low-grade inflammation with hemoglobin concentration and $\mathrm{HbA} 1 \mathrm{c}$ variability in uncontrolled T2DM. We retrospectively analyzed the hematologic and metabolic clinical laboratory data for placebo groups from 10 clinical studies that were conducted between 2001 and 2010. These studies included both healthy subjects and individuals in progressive stages of metabolic disease that presented with increased chronic low-grade inflammation coincident with elevated BMI that included dyslipidemic, IGT, and T2DM participants with uncontrolled HbA1c.

With an understanding of the variability associated with progressive adiposity, inflammation, and metabolic disease, we assessed the activity of HE3286 to decrease obesityinduced inflammation and insulin resistance in T2DM.

\section{Subjects and Methods}

2.1. Studies. This paper reports the activity of HE3286 in T2DM patients with uncontrolled HbA1c. High metabolic and hematologic laboratory value variances were observed in these patients. For comparison, similar parameters were retrospectively analyzed from placebo subjects enrolled in 10 clinical studies conducted by Harbor Therapeutics, Inc. (formerly Hollis-Eden Pharmaceuticals, Inc.) since 2001. These studies included healthy volunteers, dyslipidemic, IGT, and T2DM participants. Only placebo subjects from these studies were used for intercomparison. All studies excluded patients with known liver disease and alcoholism. The protocols and all amendments were reviewed and approved by the relevant institutional review boards, and all studies were conducted in accordance with the Declaration of Helsinki and the International Conference on Harmonization/WHO Good Clinical Practice Standards. Experimental studies were conducted with the understanding and informed consent of human subjects.

Details of studies 2100-200, -201, -202, and -203 have been published [20]. These four double-blind, randomized, placebo-controlled, healthy human safety studies were conducted in The Netherlands (Kendle International, Utrecht) and the United States (Parexel International, Baltimore, MD). Two single-dose, dose-escalation studies assured safety and evaluated the pharmacokinetics of androst-5-ene- $3 \beta, 17 \beta$ diol (HE2100) (studies 200 and 202). A multidose, dose escalation study was performed to assess safety and pharmacokinetics and potential early activity of HE2100 (study 
201). Early activity, defined by effects on peripheral blood elements, was confirmed by a follow-up study that included elderly subjects and an initial study of bone marrow hematology (study 203).

Details of studies 2200-100,-101,-120, and -130 have also been published [21]. Healthy adult and elderly subjects were randomized to receive three consecutive daily subcutaneous injections of placebo, 50 , or $100 \mathrm{mg}$ androst-5-ene$3 \beta, 7 \beta, 17 \beta$-triol (HE2200), followed by 2 months of periodic observation (trial 2200-100), or to receive placebo, 25 , or $100 \mathrm{mg}$ HE2200 transmucosally (buccal administration) once daily for five days followed by 2 months of periodic observation (trial 2200-101). Study 2200-120 was a phase II study in healthy hepatitis B-naïve, and elderly (65-85 years old) volunteers, who received hepatitis B vaccine, were randomized to concomitantly receive either $100 \mathrm{mg}$ of HE2200 or placebo equivalent. Subjects received three subcutaneous injections of study drug or placebo prior to the first and second doses of hepatitis B vaccine given 28 days apart. The third dose of vaccine was given at 6 months without HE2200 or placebo treatment, and the study terminated 28 days later.

Study 2200-130 was a phase II study in dyslipidemic subjects, ages 18-70 years, with plasma triglyceride concentrations $1.7-2 \mathrm{mmol} / \mathrm{L}$, total cholesterol levels of $5.7-8.3 \mathrm{mmol} / \mathrm{L}$, and HDL levels of $\leq 1.2 \mathrm{mmol} / \mathrm{L}$ for males and $\leq 1.4 \mathrm{mmol} / \mathrm{L}$ for females. After informed consent was obtained, subjects initiated a Step II AHA diet and discontinued all lipid lowering agents for a six-week run-in period. Each subject's lipid profile at week four of the diet was used to determine eligibility for the study. At six weeks, qualified subjects were randomized to receive 25 or $100 \mathrm{mg}$ of HE2200 or placebo equivalent by buccal administration for 28 days.

HE3286-0102 was a multicenter, double-blind, doseranging phase I study designed with 5 cohorts of obese, impaired glucose tolerance (IGT), but otherwise healthy participants [9]. Subjects were screened for fasting blood glucose level of $<7.0 \mathrm{mmol} / \mathrm{L}$ and a $7.8-11.1 \mathrm{mmol} / \mathrm{L} 2$-hour postprandial glucose following a 75-gram oral glucose load. Oral placebo or HE3286 doses of 4 (2 BID), 5 (QD), 10 (5 BID), and 20 (10 BID) mg were administered daily for 28 days. One-step hyperinsulinemic, euglycemic clamps were performed on the day before the first dose and day 29 in the BID dose groups.

For comparisons, the number of placebo subjects was 70 healthy, 24 dyslipidemic, 13 IGT, 28 treatment-naïve, and 38 metformin-treated T2DM.

2.2. Placebo Data Analysis. Placebo data from 10 clinical studies was analyzed to obtain coefficients of variation (CV) for red blood cells (RBC), hematocrit (Hct), mean corpuscular volume $(\mathrm{MCV})$, mean corpuscular hemoglobin $(\mathrm{MCH})$, mean corpuscular hemoglobin concentration (MCHC), hemoglobin (Hb), hemoglobin A1c (HbA1c), platelets, lymphocytes, monocytes, white blood cells, fasting glucose, insulin, triglycerides, cholesterol, high-density lipoprotein cholesterol (HDL), and low-density lipoprotein cholesterol (LDL). CVs from each medical condition were compared to those of healthy volunteers for significant differences in magnitude using Welch ANOVA, allowing for unequal differences, and for significant differences in dispersion, using the 2-sided $F$ test. Only placebo values were used to avoid any treatment effects from drug administrations. Pearson correlations between individual subject CVs for each parameter and CVs for $\mathrm{HbAlc}$ and for insulin were determined to assess commonality in increased variances between parameters. In addition, intravisit changes in $\mathrm{HbA1c}$ were determined for each subject with $\mathrm{HbAlc}$ data.

2.3. HE3286-0401 Data Analysis. Data for all placebo- and HE3286-treated T2DM subjects were analyzed for the same parameters by cohort (metformin treated and treatment naïve) and in subgroups (stratified by baseline monocyte chemoattractant protein-1 (MCP-1) in metformin-treated subjects, and by body mass index (BMI) in the MCP-1 selected treatment-naïve subjects). Baseline characteristics were assessed for balance between groups.

Based on the findings of excessive variability in T2DM, including random effects in intravisit $\mathrm{Hb}, \mathrm{HbA} 1 \mathrm{c}$ values were normalized ( $\mathrm{nHbA1c}$ ) by the 84 day average $\mathrm{Hb}$ value for each subject. This was done based on the fact that random effects have a mean of zero, but an excessive dispersion. Thus the average change in placebo subjects over 84 days would be expected to be zero, and true changes in HbAlc due to treatment could be compared.

Correlations were determined for independent variables of baseline MCP-1, baseline tumor necrosis factor alpha $(\mathrm{TNF} \alpha), \mathrm{BMI}, \mathrm{Hb} \mathrm{CV}$, and day 84 changes in homeostatic model assessment of beta cell function (HOMA2 \%B), glucose, weight, $\mathrm{Hb}, \mathrm{TNF} \alpha$, and MCP-1 with dependent variables $\mathrm{Hb} \mathrm{CV}, \mathrm{HbA1c} \mathrm{CV}$, and changes in $\mathrm{HbA} 1 \mathrm{c}$ and 84 day average normalized $\mathrm{HbA1c}(\triangle \mathrm{nHbA} 1 \mathrm{c})$, in order to understand clinical parameters affecting variability and treatment effects. The magnitude of HE3286 treatment effects compared to placebo was tested in the subgroups of MCP-1 $>40 \mathrm{pg} / \mathrm{mL}$ in metformin-treated subjects, and of BMI > 31 in treatment-naïve subjects.

Heteroscedasticity (differences in variances between subgroups) was tested for changes in insulin, C-peptide, fasting glucose, HOMA2 \%B and HOMA2 insulin resistance (HOMA2 IR), leptin, HbA1c, insulin, MCP-1, and triglycerides. Subgroup distributions were tested for normality (Shapiro-Wilks $W$ test) for HE3286 and placebo treatment. Differences in dispersions between HE3286 and placebo treatment were analyzed using the 2 -sided $F$ test.

2.4. Test Article HE3286. HE3286, 17 $\alpha$-ethynylandrost-5ene-3 $\beta, 7 \beta, 17 \beta$-triol active pharmaceutical ingredient was manufactured by Norac, Azuza, CA, and formulated and filled in gelatin capsules by Eminent Services Corporation, Frederick, MD. All manufacturing procedures were performed according to current good manufacturing practices.

2.5. Study HE32866-0401. The phase II trial design was a double-blind, randomized, placebo-controlled parallel group study of the safety, tolerance, and activity of HE3286 when 


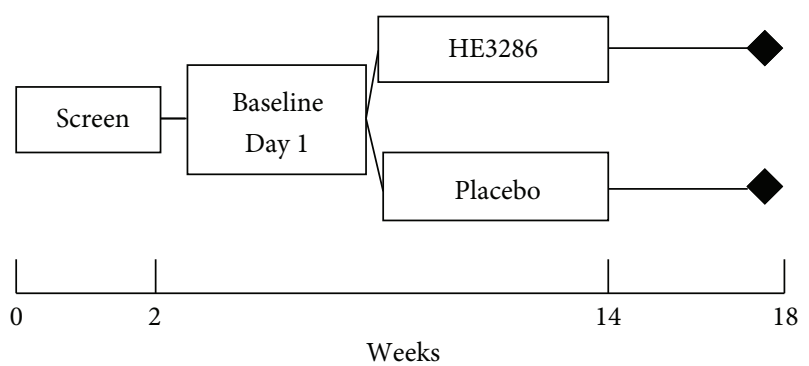

(a)

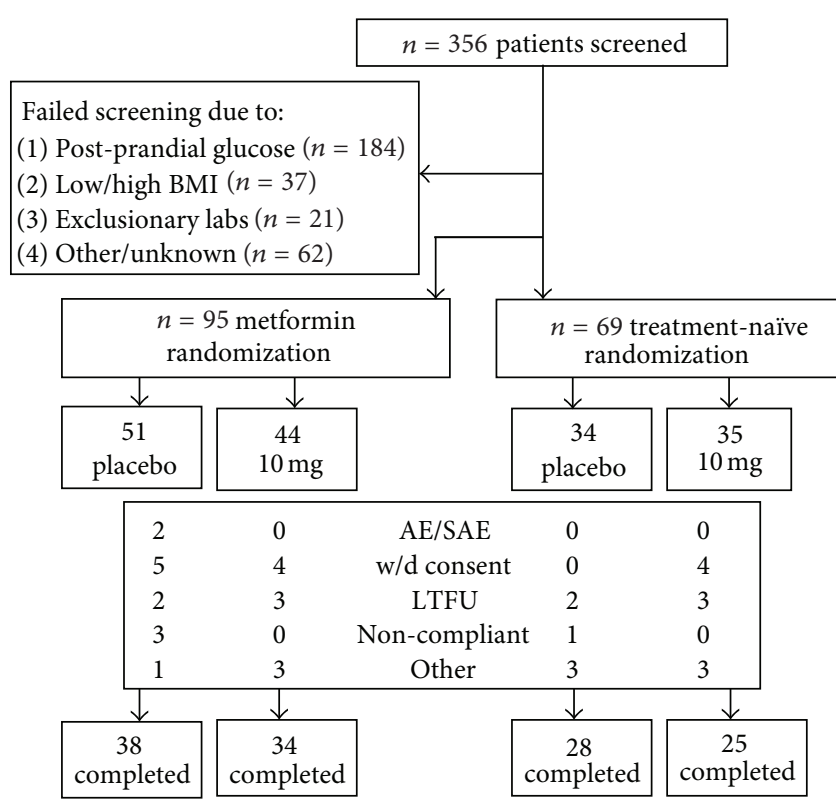

(b)

Figure 1: Study HE3286-0401 in type 2 diabetic subjects. HE32860401 study design (a) and study flow and numbers (b) for each cohort. BMI: body mass index, w/d: withdrew, and LTFU: lost to followup.

administered orally for 12 weeks to adult T2DM patients (Figure 1(a)). This was an adaptive design to investigate the characteristics of T2DM subjects that respond to HE3286. In cohort 1 of the study, 95 eligible patients, who consented to participate, were randomized $1: 1$ to receive study treatment (HE3286 $10 \mathrm{mg} /$ day or placebo) in addition to a stable dose of metformin. Inclusion criteria for cohort 1 included $\mathrm{HbAlc}$ $\geq 7.5 \%$ and fasting glucose $\leq 12.5 \mathrm{mmol} / \mathrm{L}$. In cohort 2,69 subjects who consented to participate and who met a revised eligibility criteria as determined by cohort 1 were randomized $1: 1$ to receive study treatment (HE3286 $10 \mathrm{mg}$ /day or placebo) as monotherapy. After the analysis of data from the first stage of the study, the population for cohort 2 was phenotypically enriched by screening for the following: HbA1c $7.0-10.5 \%$, fasting glucose $\leq 12.5 \mathrm{mmol} / \mathrm{L}, \mathrm{BMI} \geq$ $28 \mathrm{~kg} / \mathrm{m}^{2}$, insulin $\geq 27.8 \mathrm{pmol} / \mathrm{L}$, C-peptide $\geq 0.67 \mathrm{nmol} / \mathrm{L}$, and serum MCP- $1 \geq 36 \mathrm{pmol} / \mathrm{L}$. Subjects were screened and enrolled through outpatient clinics. The sponsor selected sites after a site visit to determine site qualifications and the investigator's ability to conduct clinical investigations according to the protocol and current Good Clinical Practice regulations: clinical trial registration: HE3286-0401 NCT00694057 http://www.clinicaltrials.gov.

\subsection{Analysis of Variance in Erythroid and Metabolic Param-} eters in Placebo Comparison Studies. Variance in erythroid parameters was examined by three ways. First, the variances for selected hematologic and metabolic laboratory values, such as the mean coefficient of variation $(\mathrm{CV})$ and the $\mathrm{CV}$ range for each individual subject, were determined and compared with those of healthy subjects. Second, the intravisit changes in $\mathrm{HbAlc}$ were compared for individual subjects for each condition with those of healthy subjects. Third, intravisit and day 84 changes in $\mathrm{HbAlc}$ and other hematology and laboratory parameters were tested for random effects.

2.7. Statistical Analyses. Random effects were tested using Residual Maximum Likelihood (REML) using StatXact, and outliers were examined using Mahalanobis distance (Cytel Software Corporation, Cambridge, MA) in conjunction with SAS software (SAS Institute, Cary, NC). Correlations were tested using Spearman or Pearson correlations, and the hypothesis that placebo participants with clinical conditions have higher frequencies of abnormal hematology and laboratory values than healthy subjects was tested using onetailed Fisher's exact test. Heteroscedasticity (tests of different variabilities between subpopulations) was tested for normal distributions (Shapiro-Wilks $W$ test), and dispersion was tested using the 2-sided $F$ test (Prism Graph Pad, San Diego, $\mathrm{CA}$ ). If there were significant differences in variances between groups, they were further examined using a $t$-test assuming unequal variances, nonparametric Mann-Whitney test, or Fisher's exact test. Due to the exploratory nature of this hypothesis-testing study, $P$ values were not adjusted for multiple comparisons.

2.8. Normalization of HbA1c to Remove Random Effects in HE3286-0401. Through the course of this analysis it was discovered that the inflammatory status of the selected patient population created large and rapid changes in the patient's red cell mass that affected the whole body hemoglobin mass and consequently the fidelity of the HbAlc metric. In order to investigate HE3286 treatment effects on HbA1c in T2DM patients, HbA1c changes were normalized to the day 84 average $\mathrm{Hb}$ for each subject, by averaging $\mathrm{Hb}$ values acquired at each clinic visit. This is statistically justified based on the fact that random effects have a mean of zero but are characterized with high variances. Normalized HbAlc (nHbAlc) was applied to correct for the inflammationinduced variances found in this T2DM study population with uncontrolled inflammation.

2.9. Details of Normalization of HbA1c Using 84-Day Average Hemoglobin in HE3286-0401. HbA1c is reported in units of $\%$ hemoglobin $(\% \mathrm{Hb}) . \mathbf{H b}$ is the concentration of hemoglobin (reported in units of $\mathrm{g} / \mathrm{dL}$ ). blood volume (male) $=0.6041+0.3669 *$ (height in meters) ${ }^{3}+0.03219 *$ (weight in $\mathrm{kg})$. blood volume $($ female $)=0.1833+0.3561 *$ (height in 
meters $)^{3}+0.03308 *($ weight in $\mathrm{kg}) .($ Blood volume unit $=\mathrm{L})$. total $\mathbf{H b}$ mass $=\mathbf{H b} * 10 *$ blood volume $(\mathrm{Hb}$ mass units $=\mathrm{g})$. total $\mathrm{HbA1c}=\mathrm{HbA1} \mathbf{c} *$ total $\mathbf{H b}$ mass (total HbA1c units = g). 84 day average total $\mathrm{Hb}$ mass = mean of baseline to day 84 total $\mathrm{Hb}$ mass measurements.

Normalized HbA1c $($ nHbA1c $)=100 *($ total HbA1c/84

day average total $\mathrm{Hb}$ mass $)(\mathrm{nHbA} 1 \mathrm{c}$ units $=\% \mathrm{Hb})$.

$\triangle$ nHbAlc represents change in $\mathbf{n H b A 1 c}$.

day $84 \Delta \mathrm{nHbA1c}=$ day $84 \mathrm{nHbA1c}-$ baseline $\mathrm{nHbA1c}$.

\section{Results}

3.1. Retrospective Exploration of Increased Variance with Metabolic Disease Progression. The hypothesis that chronic low-grade inflammation leads to increased variance in laboratory values was explored by a retrospective review of hematology and metabolic clinical parameters from placebo subjects enrolled in 10 clinical studies conducted by Harbor Therapeutics, Inc., since 2001. Only placebo subject data from these studies were used for intercomparisons to exclude study drug effects.

3.2. Variances of Hematology and Laboratory Values between Medical Conditions in Placebo Subjects. Changes in variance (CV means and ranges) for hematologic and metabolic parameters sorted by medical condition are displayed in Figure 2. Dyslipidemic patients showed increased variances in hematocrit, $\mathrm{HbAlc}$, and fasting glucose compared to healthy subjects. Although their lipid parameters were abnormal, their lipid variances were not significantly higher than those of healthy subjects. IGT subjects had significantly higher variances for RBC, hematocrit, mean corpuscular hemoglobin, mean corpuscular hemoglobin concentration, hemoglobin, and HbA1c. Although they had higher postprandial glucose, their fasting glucose variances were not significantly greater than those of healthy subjects. Metformintreated T2DM patients had significantly higher variances for RBC, hematocrit, hemoglobin, HbA1c, platelets, fasting glucose, cholesterol, and LDL. Treatment-naïve T2DM patients had significantly elevated variances for RBC, hematocrit, mean cell volume, mean corpuscular hemoglobin, hemoglobin, $\mathrm{HbA1c}$, platelets, fasting glucose, cholesterol, HDL and LDL.

3.3. Intravisit HbA1c Changes in Placebo Subjects. Individual subject intravisit $\mathrm{HbA1c}$ changes are presented by medical condition in Figure 3. Healthy subjects $\mathrm{HbA} 1 \mathrm{c}$ values were only collected in study 2100-202. Over 28 days, the 8 subjects showed an intravisit median change of 0 and a range from -0.2 to $0.2 \% \mathrm{Hb}$, consistent with literature reports. HbAlc was measured in dyslipidemic patients on days 1 and 28, yielding a single intravisit value for 21 patients with a median of 0 and an increased range of -0.5 to $0.5 \% \mathrm{Hb}$. Subjects with dysregulated glucose showed a median change of 0 with increased ranges: IGT over 56 days ( -0.3 to $0.4 \%$ $\mathrm{Hb}$ ), metformin T2DM over 112 days (-2.2 to $2.0 \% \mathrm{Hb})$, and treatment-naïve T2DM over 112 days ( -3.4 to $2.8 \%$ $\mathrm{Hb})$. Figure 3(f) shows the intravisit changes in $\mathrm{HbAlc}$
TABLE 1: Correlations with individual treatment-naïve T2DM $\mathrm{HbAlc}$ and insulin coefficients of variation.

\begin{tabular}{lccc}
\hline HbAlc CV $^{\text {a }}$ & $n$ & Pearson correlation & $P$ value \\
\hline Hemoglobin CV & 28 & 0.44 & 0.018 \\
MCP-1 CV & 20 & 0.44 & 0.0495 \\
Glucose CV & 28 & 0.58 & 0.0009 \\
C-reactive protein CV & 27 & 0.39 & 0.045 \\
HDL CV & 27 & 0.68 & $<0.0001$ \\
LDL CV & 22 & 0.52 & 0.013 \\
Triglyceride CV & 28 & 0.62 & 0.0004 \\
Lymphocyte CV & 26 & 0.44 & 0.0261 \\
Monocyte CV & 27 & 0.56 & 0.0026 \\
Platelet CV & 28 & 0.42 & 0.025 \\
Red blood cell CV & 28 & 0.53 & 0.0036 \\
Hematocrit CV & 28 & 0.48 & 0.0094 \\
MCV CV & 28 & 0.38 & 0.048 \\
\hline Insulin CV & $n$ & Pearson correlation & $P$ value \\
\hline Glucose CV & 28 & 0.46 & 0.013 \\
White blood cell CV & 28 & 0.46 & 0.013 \\
Neutrophil CV & 28 & 0.54 & 0.0028
\end{tabular}

AbA1c: hemoglobin A1c; CV: coefficient of variation; MCP-1: monocyte chemoattractant protein 1; HDL: high-density lipoprotein; LDL: low-density lipoprotein; MCV: mean corpuscular volume.

for each medical condition on the same scale. Intravisit HbA1c changes for individual T2DM patients showed 10fold increases over healthy subjects. These results indicate that the intravisit variances for individual T2DM patients are increased and distinct from the normal variances in healthy subjects.

3.4. Correlations with $H b A 1 c$ and Insulin Variance in Treatment-Naïve Placebo Subjects. Treatment-naïve T2DM patients had the greatest variance in $\mathrm{HbA1c}$. In this group, Pearson correlations were used to investigate the $\mathrm{HbAlc}$ and insulin variance relationships with other clinical parameter variances (Table 1). Individual patient $\mathrm{HbA1c} C V$, were correlated with CVs for hemoglobin, MCP-1, glucose, CRP, HDL, LDL, triglycerides, lymphocytes, monocytes, platelets, $\mathrm{RBC}$, hematocrit, and MCV, and insulin CVs were similarly correlated with CVs for glucose, WBC, and neutrophils, indicating dysregulation of multiple hematopoietic and metabolic functions within the same individual. Furthermore, REML analyses demonstrated significant random changes in day 84 glucose $(P<0.0001)$, hemoglobin $(P<0.0001)$, HbAlc $(P=$ $0.02)$, and HOMA2 \%B $(P=0.004)$, indicating laboratory results unreflective of the clinical situation.

3.5. Conclusions from the Retrospective Analysis of Placebo Subjects with Medical Conditions. There appears to be a progressive increase in metabolic and hematologic laboratory parameter variances with increased BMI and metabolic disease progression that results in random $\mathrm{HbA1c}$ changes. Based on this, intervention with the HE3286 antiinflammatory agent might confer benefit to this pathology. However, with random HbAlc effects in the placebo 
$\mathrm{RBC}$

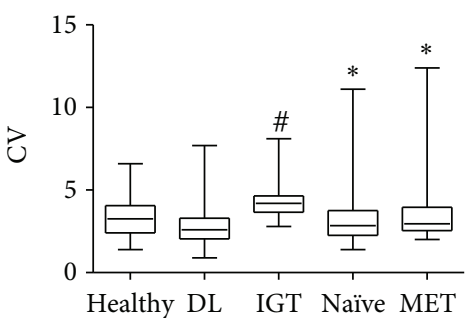

$\mathrm{MCH}$

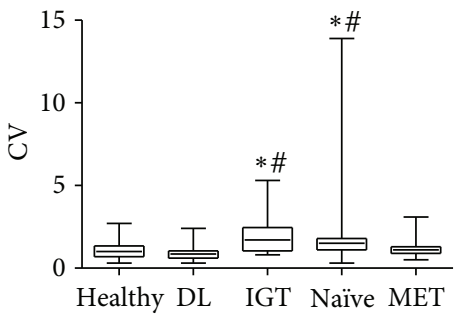

HbAlc

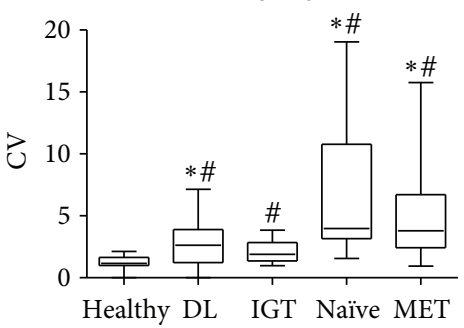

Cholesterol

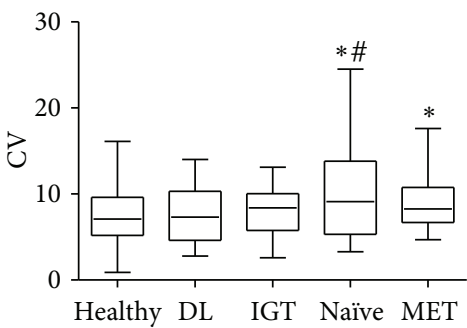

Hct

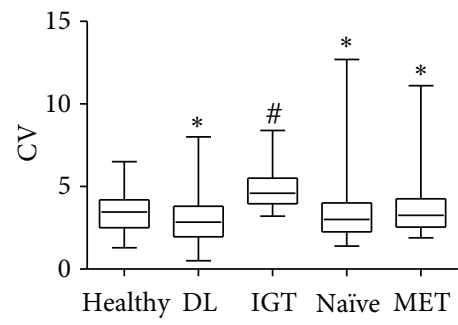

MCHC

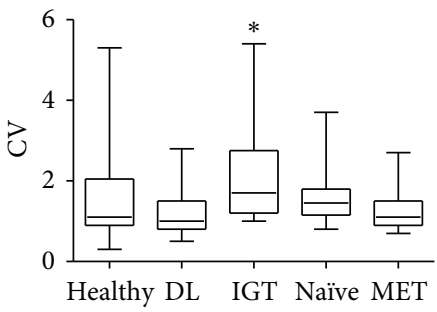

Platelets

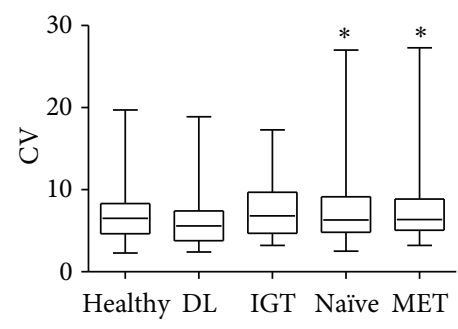

HDL

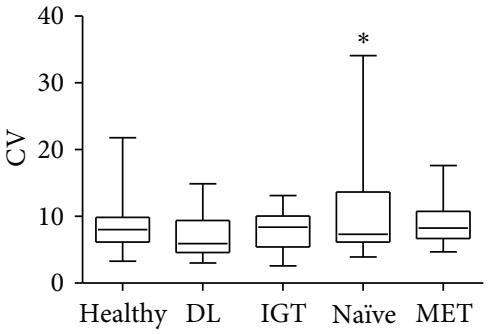

MCV

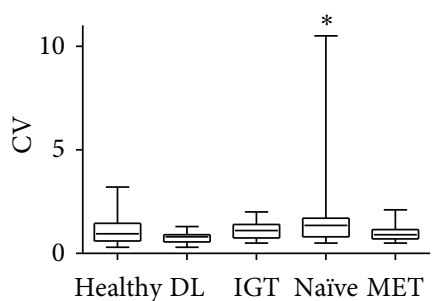

$\mathrm{Hb}$

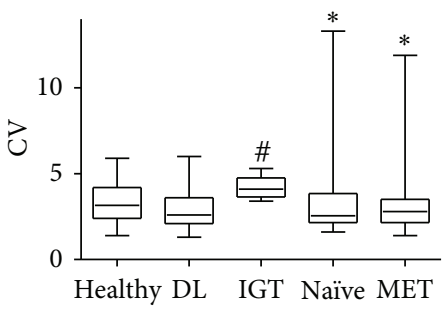

Fasting glucose

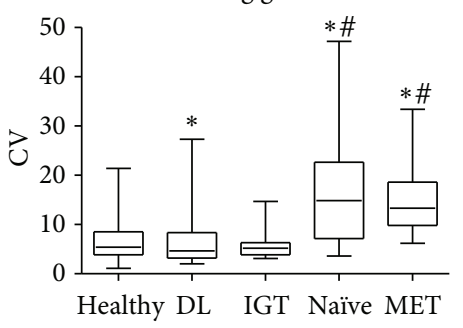

LDL

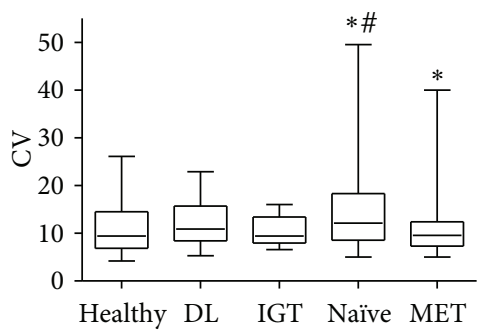

FIGURE 2: Coefficients of variation for individual subject hematology and chemistry values in placebo comparisons. DL: dyslipidemic placebo group, IGT: impaired glucose tolerant placebo group, Naïve: treatment-naïve uncontrolled T2DM placebo group, MET: uncontrolled T2DM placebo group on a stable dose of metformin, Hb: hemoglobin, Hct: hematocrit, RBC: red blood cell count, MCV: mean cell volume, $\mathrm{MCH}$ : mean corpuscular hemoglobin, MCHC: mean corpuscular hemoglobin concentration, HbA1c: hemoglobin A1c, HDL: high density lipoprotein cholesterol, LDL: low density lipoprotein cholesterol. ${ }^{\#}$ Statistically significant (Welch ANOVA, allowing unequal variance) increase in mean coefficient of variation compared to healthy participants. ${ }^{*}$ Statistically significant dispersion $(2$-sided $F$ test $)$ in the coefficient of variation compared to healthy participants.

participants, it is difficult to demonstrate significant changes with comparisons to active agents unless a correction is applied to the data. Accordingly, treatment effects were investigated by normalizing $\mathrm{HbAlc}$ to the day 84 average total body hemoglobin mass for each patient.

3.6. HE3286-0401. In this study, HE3286 was well tolerated. Seventy-six percent of cohort 1 (metformin treated) and $77 \%$ of cohort 2 (treatment naïve) completed the study (Figure 1(b)). Only 1 serious adverse event occurred, a transient asymptomatic elevation from baseline of blood amylase, which resolved on study. This event was considered by the investigator to be possibly related to study medication. There were no clinically significant abnormalities related to any body system, including hypoglycemia and electrocardiograms, attributable to HE3286 administration. There were no detectable differences or trends in adverse events between placebo- and HE3286-treated subjects. No patient died while on study. Baseline demographics and characteristics of each group are presented in Table 2. 
TABLE 2: HE3286-0401 baseline demographics and characteristics of each group.

\begin{tabular}{|c|c|c|c|c|c|c|}
\hline \multirow{2}{*}{ Cohort 1} & \multicolumn{2}{|c|}{ All Subjects } & \multicolumn{2}{|c|}{$\mathrm{MCP} 1^{\mathrm{a}}>40 \mathrm{pmol} / \mathrm{L}$} & \multicolumn{2}{|c|}{$\mathrm{MCP}-1 \leq 40 \mathrm{pmol} / \mathrm{L}$} \\
\hline & HE3286 & Placebo & HE3286 & Placebo & HE3286 & Placebo \\
\hline$n$ & 34 & 38 & 22 & 25 & 12 & 13 \\
\hline Age & $48(41-57)^{\mathrm{b}}$ & $50(43-59)$ & $49(40-57)$ & $12(44-61)$ & $48(41-57)$ & $48(43-56)$ \\
\hline Gender F (\%) & $19(56 \%)$ & $18(47 \%)$ & $12(56 \%)$ & $12(48 \%)$ & $7(58 \%)$ & $6(46 \%)$ \\
\hline \multicolumn{7}{|l|}{ Ethnicity } \\
\hline White/White Hispanic & $25(74 \%)$ & $27(71 \%)$ & $18(82 \%)$ & $18(72 \%)$ & $7(58 \%)$ & $9(69 \%)$ \\
\hline Black & $5(15 \%)$ & $7(18 \%)$ & $2(9 \%)$ & $3(12 \%)$ & $3(25 \%)$ & $4(31 \%)$ \\
\hline Asian & $3(9 \%)$ & $3(8 \%)$ & $1(5 \%)$ & $3(12 \%)$ & $1(8 \%)$ & $0(0 \%)$ \\
\hline Other & $1(3 \%)$ & $1(3 \%)$ & $1(5 \%)$ & $1(4 \%)$ & $1(8 \%)$ & $0(0 \%)$ \\
\hline MCP-1 (pmol/L) & $50(35-87)$ & $52(34-70)$ & $61(50-75)$ & $61(52-81)$ & $32(27-36)$ & $18(20-36)$ \\
\hline $\mathrm{TNF} \alpha(\mathrm{pmol} / \mathrm{L})$ & $0(0-0.1)$ & $0(0-0.3)$ & $0(0-0.1)$ & $0(0-0.4)$ & $0(0-0)$ & $0(0-0.4)$ \\
\hline BMI $\left(\mathrm{kg} / \mathrm{m}^{2}\right)$ & $29(25-32)$ & $30(26-36)$ & $29(26-32)$ & $29(25-37)$ & $27(25-31)$ & $33(29-36)$ \\
\hline $\mathrm{HbAlc}(\% \mathrm{Hb})$ & $8.5(7.8-9.3)$ & $8.3(8.0-9.2)$ & $9.0(7.9-9.6)$ & $8.3(8.0-9.3)$ & $8.0(7.8-8.6)$ & $8.5(8.0-9.2)$ \\
\hline $\mathrm{Hb}(\mathrm{mmol} / \mathrm{L})$ & $8.5(8.1-9.3)$ & $8.5(8.1-9.3)$ & $8.6(8.1-9.3)$ & $8.3(8.1-9.3)$ & $8.0(7.5-8.7)$ & $9.1(8.1-9.3)$ \\
\hline Hct (proportion) & $0.41(0.37-0.43)$ & $0.41(0.39-0.44)$ & $0.41(0.39-0.44)$ & $0.40(0.39-0.43)$ & $0.39(0.36-0.42)$ & $0.44(0.39-0.46)$ \\
\hline $\operatorname{RBC}\left(10^{12} / \mathrm{L}\right)$ & $4.6(4.3-4.9)$ & $4.7(4.4-4.9)$ & $4.6(4.4-5.0)$ & $4.7(4.4-5.0)$ & $4.6(4.3-4.9)$ & $4.7(4.4-5.1)$ \\
\hline Insulin (pmol/L) & $56(28-90)$ & $63(35-118)$ & $69(29-97)$ & $56(28-104)$ & $56(42-76)$ & $90(35-188)$ \\
\hline C-peptide (nmol/L) & $0.77(0.60-1.0)$ & $0.90(0.53-1.2)$ & $0.80(0.50-1.1)$ & $0.70(0.53-1.1)$ & $0.73(0.67-0.87)$ & $1.0(0.53-1.4)$ \\
\hline Fasting glucose (mmol/L) & $8.9(7.7-11)$ & $9.2(7.5-10.1)$ & $9.5(8.3-11)$ & $8.4(7.5-10)$ & $8.1(7.5-8.7)$ & $9.2(7.2-10)$ \\
\hline 1,5-Anhydroglucitol $(\mu \mathrm{mol} / \mathrm{L})^{\mathrm{c}}$ & $35(12-60)$ & $52(20-85)$ & & & & \\
\hline $\mathrm{CRP}(\mathrm{pmol} / \mathrm{L})$ & $30(14-61)$ & $25(12-73)$ & $29(12-49)$ & $13(11-73)$ & $35(18-94)$ & $26(15-57)$ \\
\hline HOMA2 \%B & $47(36-62)$ & $59(33-82)$ & $41(33-57)$ & $58(30-80)$ & $58(47-67)$ & $70(37-88)$ \\
\hline HOMA2 IR & $2.0(1.5-2.7)$ & $2.2(1.4-3.2)$ & $2.3(1.3-2.9)$ & $2.0(1.4-2.9)$ & $1.9(1.6-2.3)$ & $2.8(1.3-3.6)$ \\
\hline Triglycerides $(\mathrm{mmol} / \mathrm{L})$ & $1.8(1.3-2.9)$ & $2.0(1.3-2.5)$ & $1.7(1.2-3.1)$ & $2.1(1.3-2.7)$ & $2.0(1.4-2.6)$ & $2.0(1.3-2.1)$ \\
\hline \multirow{2}{*}{ Cohort 2} & \multicolumn{2}{|c|}{ All subjects } & \multicolumn{2}{|c|}{$\mathrm{BMI}>31 \mathrm{~kg} / \mathrm{m}^{2}$} & \multicolumn{2}{|c|}{$\mathrm{BMI} \leq 31 \mathrm{~kg} / \mathrm{m}^{2}$} \\
\hline & HE3286 & Placebo & HE3286 & Placebo & HE3286 & Placebo \\
\hline$n$ & 25 & 28 & 12 & 15 & 13 & 13 \\
\hline Age & $54(48-60)$ & $53(46-58)$ & $53(42-56)$ & $53(46-55)$ & $55(49-63)$ & $58(46-62)$ \\
\hline Gender F (\%) & $10(40 \%)$ & $14(50 \%)$ & $4(33 \%)$ & $9(60 \%)$ & $6(46 \%)$ & $5(38 \%)$ \\
\hline \multicolumn{7}{|l|}{ Ethnicity } \\
\hline White/White Hispanic & $20(80 \%)$ & $27(96 \%)$ & $10(83 \%)$ & $15(100 \%)$ & $10(77 \%)$ & $12(92 \%)$ \\
\hline Black & $3(12 \%)$ & $0(0 \%)$ & $2(17 \%)$ & $0(0 \%)$ & $1(8 \%)$ & $0(0 \%)$ \\
\hline Asian & $2(8 \%)$ & $1(4 \%)$ & $0(0 \%)$ & $0(0 \%)$ & $2(15 \%)$ & $1(8 \%)$ \\
\hline Other & $0(0 \%)$ & $0(0 \%)$ & $0(0 \%)$ & $0(0 \%)$ & $0(0 \%)$ & $0(0 \%)$ \\
\hline BMI $\left(\mathrm{kg} / \mathrm{m}^{2}\right)$ & $31(29-33)$ & $31(29-37)$ & $33(32-36)$ & $36(32-37)$ & $29(28-30)$ & $29(28-30)$ \\
\hline MCP-1 $(\mathrm{pmol} / \mathrm{L})^{\mathrm{d}}$ & $108(51-189)$ & $94(64-117)$ & $97(49-182)$ & $94(62-129)$ & $110(68-195)$ & $95(67-123)$ \\
\hline $\mathrm{HbA1c}(\% \mathrm{Hb})$ & $8.1(7.5-8.7)$ & $8.5(7.7-10.2)$ & $7.6(8.0-8.4)$ & $8.4(7.5-9.8)$ & $8.4(7.5-8.8)$ & $9.4(8.1-10.6)$ \\
\hline $\mathrm{Hb}(\mathrm{mmol} / \mathrm{L})$ & $9.4(8.7-9.9)$ & $8.8(8.1-9.9)$ & $9.5(8.7-9.9)$ & $8.9(8.1-9.9)$ & $8.9(8.7-9.9)$ & $8.8(8.1-9.9)$ \\
\hline Hct (proportion) & $0.45(0.41-0.46)$ & $0.42(0.39-0.46)$ & $0.45(0.43-0.46)$ & $0.44(0.39-0.46)$ & $0.42(0.40-0.47)$ & $0.42(0.38-0.46)$ \\
\hline $\operatorname{RBC}\left(10^{12} / \mathrm{L}\right)$ & $4.7(4.5-5.1)$ & $4.7(4.5-5.1)$ & $4.9(4.6-5.1)$ & $4.8(4.5-5.0)$ & $4.7(4.3-5.0)$ & $4.5(4.3-5.1)$ \\
\hline Insulin (pmol/L) & $104(69-146)$ & $111(76-153)$ & $118(76-139)$ & $118(76-146)$ & $97(69-153)$ & $104(69-174)$ \\
\hline C-peptide (nmol/L) & $1.1(0.8-1.3)$ & $1.1(0.9-1.4)$ & $1.2(0.8-1.3)$ & $1.2(1.0-1.3)$ & $1.0(0.8-1.2)$ & $1.0(0.9-1.5)$ \\
\hline Fasting glucose (mmol/L) & $8.3(7.2-9.9)$ & $9.2(7.3-11)$ & $8.0(6.7-8.8)$ & $8.2(6.8-10)$ & $8.5(7.2-11)$ & $9.3(8.1-13)$ \\
\hline 1,5-Anhydroglucitol $(\mu \mathrm{mol} / \mathrm{L})^{\mathrm{e}}$ & $54(31-83)$ & $102(35-154)$ & & & & \\
\hline $\mathrm{CRP}(\mathrm{pmol} / \mathrm{L})$ & $27(19-49)$ & $32(16-48)$ & $27(22-67)$ & $33(22-53)$ & $28(13-48)$ & $30(12-45)$ \\
\hline HOMA2 \%B & $74(43-108)$ & $61(45-89)$ & $87(47-123)$ & $80(43-120)$ & $71(35-85)$ & $56(46-73)$ \\
\hline HOMA2 IR & $2.7(2.2-3.4)$ & $2.8(2.5-3.8)$ & $2.9(2.1-3.4)$ & $3.2(2.5-3.9)$ & $2.6(2.2-3.3)$ & $2.7(2.5-4.3)$ \\
\hline Triglycerides (mmol/L) & $1.6(1.1-2.7)$ & $2.1(1.5-3.5)$ & $1.4(1.1-1.8)$ & $2.0(1.6-2.8)$ & $1.4(2.3-3.2)$ & $3.5(1.4-6.7)$ \\
\hline
\end{tabular}

MCP-1: monocyte chemoattractant protein 1; TNF $\alpha$ : tumor necrosis factor alpha; BMI: body mass index; HbA1c: hemoglobin Alc; Hb: hemoglobin; Hct: hematocrit; RBC: red blood cells; CRP: C-reactive protein; HOMA2 \%B: homeostatic model assessment of \% pancreatic beta cell function, HOMA2 IR: homeostatic model assessment of insulin resistance. ${ }^{b}$ Numbers are medians (IQR) or numbers (\%). ${ }^{c} 1,5$-Anhydroglucitol only 19 retention samples $(9 \mathrm{HE} 3286$, 10 placebo, predominantly with $\mathrm{MCP}>40 \mathrm{pmol} / \mathrm{mL}$ ). ${ }^{\mathrm{d}} \mathrm{MCP}-1$ only 38 samples (18 HE3286, 20 placebo); ${ }^{\mathrm{e}} 1,5$-Anhydroglucitol only 23 retention samples ( 9 HE3286, 14 placebo, predominantly from BMI $>31 \mathrm{~kg} / \mathrm{m}^{2}$ subjects). 


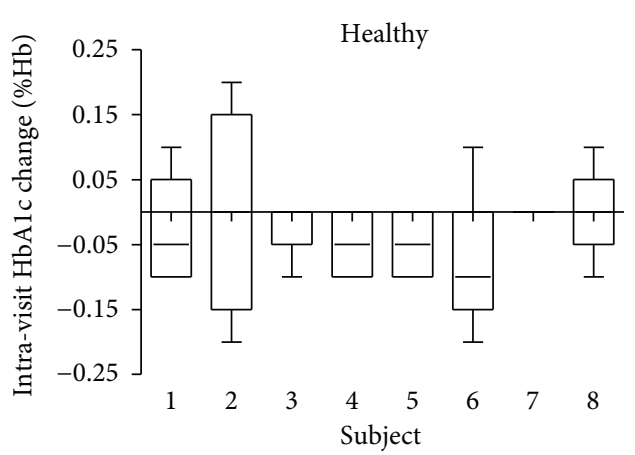

(a)

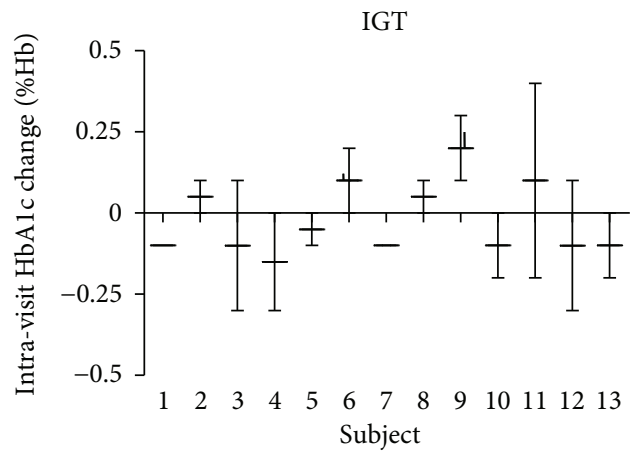

(c)

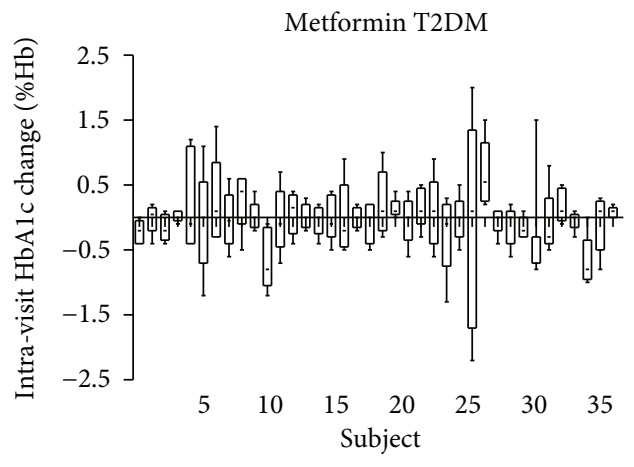

(e)

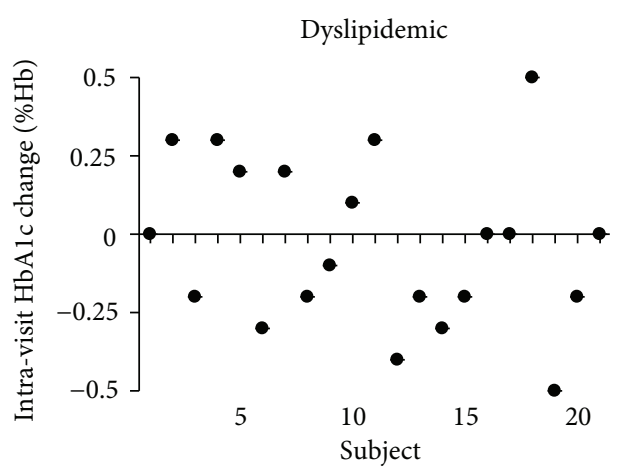

(b)

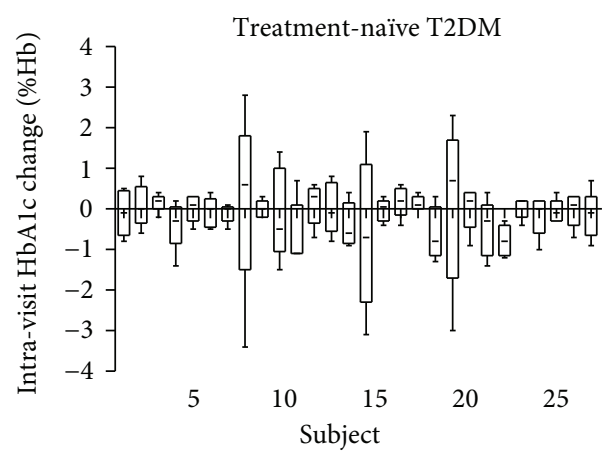

(d)

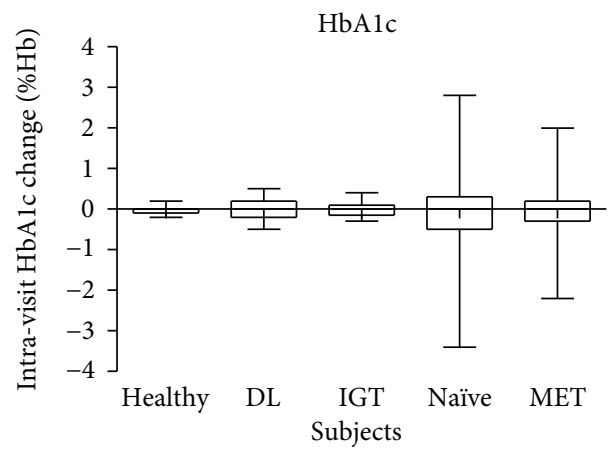

(f)

FiguRE 3: Intrasubject HbA1c changes in placebo comparisons. Bar and whisker plots of the change in HbAlc between visits for individual subjects: (a) healthy subjects from study 2100-202, (b) dyslipidemic subjects from study 2200-130, (c) impaired glucose tolerance subjects from study 3286-0102, (d) treatment-naïve type 2 diabetes patients from HE3286-0401 cohort 2, (e) type 2 diabetic patients on a stable dose of metformin from HE3286-0401 cohort 1, (f) intravisit changes for all subjects from each condition plotted on the same scale. IGT: impaired glucose tolerant, Metformin T2DM: uncontrolled type 2 diabetes mellitus participants on a stable dose of metformin.

\subsection{Correlates of HbA1c and Hemoglobin Changes and Variances in HE3286 and Placebo Subjects}

3.7.1. Cohort 1. Correlations of $\triangle \mathrm{HbA1c}, \mathrm{HbA1c} \mathrm{CV}$, and $\mathrm{Hb}$ CV with other parameters are shown in Table 3. The HE3286 cohort 1 group $\mathrm{HbA} 1 \mathrm{c}$ change was negatively correlated with baseline MCP-1 $(P=0.01)$ and change in HOMA2 \%B $(P=0.01)$ and positively correlated with glucose change $(P=$ $0.009)$, weight change $(P=0.007)$, and change in $\mathrm{Hb}(P=$ $0.03)$. HbA1c change was positively correlated with fasting glucose change in both HE3286 and placebo. The placebo
HbA1c change was negatively correlated with baseline TNF $\alpha$ $(P=0.004)$ and positively with change in $\operatorname{TNF} \alpha(P=$ $0.02)$. In addition, in the placebo group, intrapatient HbA1c coefficients of variation $(\mathrm{CV})$ were significantly correlated with baseline TNF $\alpha(P=0.002)$. These relationships led to the hypothesis that HE3286 decreased HbA1c in the more inflamed (higher MCP-1) patients, in conjunction with increased pancreatic beta cell function and weight loss, but in placebos, inflammation ( TNF $\alpha$ ) was primarily contributing to $\mathrm{HbA} 1 \mathrm{c}$ changes. The finding that $\mathrm{Hb}$ change was positively correlated with $\mathrm{HbAlc}$ change suggested the possibility that 
TABLE 3: HE3286-0401 significant correlates of HbA1c and Hb changes.

\begin{tabular}{|c|c|c|c|c|c|c|c|c|c|c|c|}
\hline \multirow{2}{*}{ Group } & \multirow{2}{*}{ Dependent } & \multirow{2}{*}{ Independent } & \multirow{2}{*}{ Test } & \multicolumn{4}{|c|}{ HE3286 } & \multicolumn{4}{|c|}{ Placebo } \\
\hline & & & & $n$ & $r$ & $95 \% \mathrm{CI}$ & $P$ & $n$ & $r$ & $95 \% \mathrm{CI}$ & $P$ \\
\hline \multirow{9}{*}{ Cohort 1} & $\Delta \mathrm{HbAlc}^{\mathrm{a}}$ & MCP-1 & Spearman & 34 & -0.42 & -0.67 to -0.08 & 0.01 & & & & $>0.10$ \\
\hline & & $\triangle \mathrm{HOMA} 2 \% \mathrm{~B}$ & Spearman & 34 & -0.43 & -0.68 to 0.10 & 0.01 & & & & $>0.10$ \\
\hline & & $\Delta$ Glucose & Spearman & 34 & 0.45 & 0.11 to 0.69 & 0.009 & & & & $>0.10$ \\
\hline & & $\Delta$ Weight & Spearman & 34 & 0.45 & 0.13 to 0.69 & 0.007 & & & & $>0.10$ \\
\hline & & $\Delta \mathrm{Hb}$ & Pearson & 34 & 0.36 & 0.03 to 0.62 & 0.03 & & & & $>0.10$ \\
\hline & $\triangle \mathrm{HbAlc}$ & $\mathrm{TNF} \alpha$ & Spearman & & & & $>0.10$ & 34 & -0.48 & -0.71 to -0.16 & 0.004 \\
\hline & & $\Delta$ Glucose & Spearman & & & & $>0.10$ & 38 & 0.65 & 0.41 to 0.81 & $<0.0001$ \\
\hline & & $\Delta \mathrm{TNF} \alpha$ & Spearman & & & & $>0.10$ & 32 & 0.40 & 0.05 to 0.66 & 0.02 \\
\hline & $\mathrm{HbA1c} \mathrm{CV}$ & $\mathrm{TNF} \alpha$ & Pearson & & & & $>0.10$ & 34 & 0.50 & 0.19 to 0.72 & 0.002 \\
\hline \multirow{7}{*}{ Cohort 2} & $\Delta \mathrm{HbA1c}$ & BMI & Spearman & 25 & -0.41 & -0.70 to -0.008 & 0.04 & & & & $>0.10$ \\
\hline & & $\triangle \mathrm{HOMA} 2 \% \mathrm{~B}$ & Pearson & 25 & -0.50 & -0.75 to -0.13 & 0.02 & & & & $>0.10$ \\
\hline & & $\Delta$ Glucose & Pearson & 25 & 0.61 & 0.29 to 0.81 & 0.001 & & & & $>0.10$ \\
\hline & $\mathrm{HbA1c} C \mathrm{CV}$ & $\mathrm{Hb} \mathrm{CV}$ & Pearson & & & & $>0.10$ & 28 & 0.44 & 0.08 to 0.70 & 0.02 \\
\hline & & $\Delta \mathrm{MCP}-1$ & Pearson & & & & $>0.10$ & $20^{\mathrm{b}}$ & 0.44 & 0.002 to 0.74 & 0.0495 \\
\hline & & $\Delta$ Weight & Pearson & & & & $>0.10$ & 28 & -0.43 & -0.69 to -0.07 & 0.02 \\
\hline & $\mathrm{Hb} \mathrm{CV}$ & MCP-1 & Spearman & & & & $>0.10$ & 20 & 0.38 & 0.03 to 0.70 & 0.04 \\
\hline \multirow{2}{*}{$\begin{array}{l}\text { Cohort } 2 \\
\text { BMI > } 31\end{array}$} & $\Delta \mathrm{nHbA1c}$ & MCP-1 & Spearman Exact & $10^{\mathrm{b}}$ & -0.68 & $--^{c}$ & 0.03 & & & & $>0.10$ \\
\hline & & $\triangle \mathrm{MCP}-1$ & Spearman Exact & $9^{b}$ & 0.77 & - & 0.002 & & & & $>0.10$ \\
\hline
\end{tabular}

$\Delta$ : change in; CV: coefficient of variation; HbA1c: hemoglobin A1c; Hb: hemoglobin; MCP-1: monocyte chemoattractant protein-1; HOMA2 \%B: homeostatic model assessment of pancreatic beta cell function; TNF $\alpha$ : tumor necrosis factor alpha; BMI: body mass index; nHbAlc: HbA1c normalized to day 84 hemoglobin mass (see Section 2 for details). ${ }^{b} \mathrm{MCP}-1$ data unavailable on a portion of participants. ${ }^{\mathrm{c}}$ Spearman Exact test has no confidence interval.

inflammation-induced effects produce random $\mathrm{Hb}$ levels that contribute to increased variance in $\mathrm{HbAlc}$ changes.

High intrapatient CVs were observed for both HbA1c (up to $16 \%$ ) and $\mathrm{Hb}$ (up to $12 \%$ ) in both $\mathrm{HE} 3286$ and placebo groups (data not shown). Residual maximum likelihood (REML) analyses indicated a significant random CV component in the intrapatient $\mathrm{Hb}$ values, for both the HE3286 and placebo groups $(P<0.0001$ for each, data not shown).

3.7.2. Cohort 2. Cohort 2 participants were selected using more stringent criteria for MCP-1, BMI, insulin, and Cpeptide. TNF $\alpha$ was not measured in this group. Consequently, significant correlations observed in cohort 1 for HbA1c change with baseline MCP-1 and changes in weight and in TNF $\alpha$ were not observed in the overall cohort 2 group. Table 3 also shows correlations between $\triangle \mathrm{HbA} 1 \mathrm{c}$, $\mathrm{HbA} 1 \mathrm{c} \mathrm{CV}$, and $\mathrm{Hb} \mathrm{CV}$ and other parameters in cohort 2. In this overweight to obese population, $\triangle \mathrm{HbA} 1 \mathrm{c}$ was correlated negatively with baseline BMI $(P=0.04)$ and with HOMA2 \% B change $(P=0.02)$ and positively with day 84 change in fasting plasma glucose $(P=0.001)$ for HE3286, but not placebo. Further, we found that the cohort 2 participants (selected for higher inflammation) had higher variances in erythroid hematology values than cohort 1 (see Figure 2), and cohort 2 placebo day 84 changes in $\mathrm{HbAlc}$ had a significant random component (REML $P=0.006$, data not shown). In placebo patients, $\mathrm{HbA1c} \mathrm{CV}$ was positively correlated with baseline $\mathrm{Hb} \mathrm{CV}(P=0.02)$ and the $\triangle \mathrm{MCP}-1$ inflammation marker $(P=0.0495)$ and negatively correlated with weight change $(P=0.02)$. $\mathrm{Hb} \mathrm{CV}$, in turn, was positively correlated with baseline MCP-1 $(P=0.04)$. These relationships led us to the hypothesis that HE3286 decreased HbA1c in patients with higher obesity (BMI), in conjunction with improved pancreatic beta cell function and decreased fasting glucose, that, for placebo patients, $\mathrm{HbAlc}$ change and $\mathrm{Hb} \mathrm{CV}$ were related to inflammation status (MCP-1), and that weight loss in placebos might be related to inflammation effects on malnutrition.

Together, the results from studies in HE3286-0401 T2DM patients suggested that low-grade chronic inflammation develops during metabolic disease progression in the obese diabetic and contributes to dysregulation of metabolic and hematologic homeostasis. If this is correct, then intervention with an anti-inflammatory compound such as HE3286 might lead to restoration of homeostasis, normalization of glucose levels, and a decline in weight. Further, the inflammatory effects on erythropoiesis may be quelled and the utility of $\triangle \mathrm{HbA} 1 \mathrm{c}$ as a biomarker of glucose control restored.

\subsection{HE3286-0401 Treatment Effects}

3.8.1. Cohort 1. According to our observations in phase I inflamed obese prediabetics, HE3286 should show benefit in inflamed T2DM individuals. The correlation of baseline MCP-1 in the HE3286 HbA1c response was explored, and significant treatment effects were observed in the more inflamed subjects (baseline serum MCP-1 upper 2 tertiles ( $>40 \mathrm{pmol} / \mathrm{L})$ ). Table 4 displays the HE3286 day 84 treatment 
effects on clinical parameters in this subgroup. Significant decreases were observed for HOMA2 IR $(P=0.02)$, Cpeptide $(P=0.04), \mathrm{Hb}(P=0.02)$, Hct $(P=0.02)$, and RBC $(P=0.02)$ changes in the HE3286 treatment group when compared to the placebo (metformin alone) group.

The effect of HE3286 on $\triangle \mathrm{nHbA1c}$ in the overall population was not significant. Therefore, the day 84 treatment effect on $\triangle \mathrm{nHbAlc}$ was investigated in the more inflamed MCP-1 subgroup (Table 4). The median magnitude of the $\triangle \mathrm{nHbA1c}$ was $-0.44 \% \mathrm{Hb}$ (HE3286, -0.34 ; placebo, +0.1 ). The HE3286 data was normally distributed, but placebo was significantly abnormal ( $P=0.0006, W$ test, data not shown). This situation necessitated the use of nonparametric methods of data analysis. The HE3286 treatment effect was found to significantly decrease nHbAlc from zero $(P=0.03)$. The frequency of HE3286 patients with decreased nHbAlc was significantly greater than placebo (17/22 versus $9 / 25$, $P=0.0008$ ). There were no significant differences between HE3286 and placebo groups at follow-up day 112.

The HE3286 and placebo patients distributions with MCP-1 $>40 \mathrm{pmol} / \mathrm{L}$ are shown for $\triangle \mathrm{nHbAlc}$ in Figure 4(a) and for $\triangle$ HOMA2 IR in Figure 4(b). The majority of HE3286 patients showed decreased nHbA1c and HOMA2 IR, whereas the majority of placebos showed increases. These results are consistent with inhibition of $\mathrm{NF} \kappa \mathrm{B}$ hyperactivation and consequent restoration of normal insulin signaling, consistent with the preclinical HE3286 observations.

3.8.2. Cohort 2. The correlation between baseline BMI and change in HbA1c in the HE3286 group was explored by stratifying participants on the median BMI $\left(31 \mathrm{~kg} / \mathrm{m}^{2}\right)$. The $\triangle \mathrm{nHbA1c}$ in the HE3286, but not placebo participants (with BMI $\left.>31 \mathrm{~kg} / \mathrm{m}^{2}\right)$, correlated significantly with their baseline MCP-1 $(P=0.03)$ (Table 3$)$. This strengthens the hypothesis that HE3286 benefited the obese inflamed subset of T2DM patients. The $\triangle \mathrm{nHbA1c}$ also correlated significantly with $\triangle$ MCP-1 $(P=0.002)$ in HE3286 (Table 3), but not in placebo participants. Thus the decrease in inflammation (MCP-1) was associated with the decrease in HbAlc with HE3286 treatment.

The effect of HE3286 on nHbA1c in the overall population was not significant. The obese patients with a BMI $\left(>31 \mathrm{~kg} / \mathrm{m}^{2}\right)$, demonstrated a significant treatment effect $(t-$ test) to decrease nHbA1c by $0.6 \% \mathrm{Hb}$ compared to placebo, but only after exclusion of 2 outliers (Mahalanobis distance). The day 84 distribution of the $\triangle \mathrm{nHbAlc}$ for BMI $>31$ is shown in Figure 4(c) (outliers circled). The variances were much higher in the treatment-naïve patients' parameters compared to uncontrolled metformin-treated patients (Figure 3(f)). We speculated that these two outliers were still subject to inflammation-induced random effects, after only 84 days of treatment and that additional treatment may be necessary to observe effects in these individuals. Because of the lag in $\triangle \mathrm{HbA} 1 \mathrm{c}$ following glucose excursions, we tested the treatment effects on follow-up day 112. A significant day 112 treatment effect (with no outliers) was found in the high BMI stratum, both by nonparametric and parametric tests (Table 4). HE3286 participants had a significant mean change from baseline $(-1.0 \% \mathrm{Hb}, P=0.0007)$, whereas placebo did not. The mean change compared to placebo was also significant $(-0.7 \% \mathrm{Hb}, P=0.03)$. The HE3286 participants also had a significant median change from baseline $(-1.2 \%$ $\mathrm{Hb}, P=0.002$ ), whereas placebo did not. The magnitude of the response in the HE3286 treatment groups was significant $(-1.0 \% \mathrm{Hb}, P=0.02)$ compared to placebo, as was the frequency of subjects with a $0.5 \%$ HbA1c decrease $(9 / 12$ versus $4 / 13, P<0.05)$. The day $112 \Delta$ nHbAlc distributions for BMI $>31 \mathrm{~kg} / \mathrm{m}^{2}$ are shown in Figure 4(d).

3.9. Postprandial Treatment Effect. A significant treatment effect that lowered fasting glucose was not found and was attributed to high metabolic parameter variations. Consequently, the possibility that HE3286 decreased HbA1c through action on postprandial glucose was investigated. Serum 1,5-anhydroglucitol (1,5-AH) is a dietary human metabolite that is reabsorbed by a kidney glucose transporter [22]. The 1,5-AH level declines when blood glucose levels are elevated above $10 \mathrm{mmol} / \mathrm{L}$ and likewise increases when the blood glucose level declines.

1,5-AH was measured in a subset of 42 participants (19 from stages 1 and 23 from stage 2) that had available day 84 retention samples. Analysis of 18 patients treated with HE3286 demonstrated that their 1,5-AH concentration increased significantly $(+10.4 \mu \mathrm{mol} / \mathrm{L}, P=0.02) ; 24$ treated with placebo demonstrated no significant concentration increase $(+0.6 \mu \mathrm{mol} / \mathrm{L}, P>0.1)$. The distribution of 1,5 $\mathrm{AH}$ responses is shown in Figure 4(e). The majority of HE3286 patients significantly increased $1,5-\mathrm{AH}$, compared to placebos (15/18 versus $11 / 24, P=0.02$, Fisher's exact test). This outcome indicates that HE3286 had a treatment effect to decrease postprandial glucose excursions compared to placebo, which further supports that it's pharmacologic property is to decrease insulin resistance (see [9] and Figure 4(b)) and lower HbAlc.

3.10. HE3286-0401 Heteroscedasticity in HE3286 and Placebo Groups. Heteroscedasticity (differences in variances between groups) was investigated by analyzing data distributions for normality (Shapiro-Wilks $W$ test) and analyzing dispersion (2-sided $F$ test). In cohort 1 placebo, but not HE3286, day 84 distributions ( $W$ test) were significantly abnormal for changes in insulin, C-peptide, fasting glucose, HOMA2 \%B, HOMA2 IR, and leptin in all subjects, and for changes in HbAlc, fasting glucose, and HOMA2 \%B for MCP-1 $>40 \mathrm{pmol} / \mathrm{L}$ participants. Variances for cohort 1 placebo subjects ( $F$ test) were also significantly higher than those of HE3286 subjects for insulin, C-peptide, and HOMA2 IR for all subjects.

Cohort 2 placebo, but not HE3286 distributions were abnormal ( $W$ test) for the group as a whole for changes in all the following parameters: day $84 \mathrm{nHbAlc}$, fasting glucose, MCP-1, and triglycerides and day $112 \mathrm{nHbA1c}$, fructosamine, and HOMA2 \%B. In the BMI > 31 subgroup, abnormal distributions were found for changes in all the following parameters: day 84 HOMA2 \%B and day 112 insulin, C-peptide, HOMA2 \%B, and HOMA2 IR. Variances 


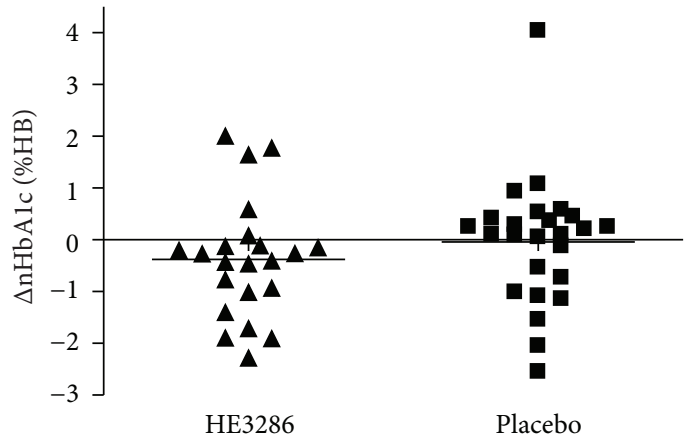

(a)

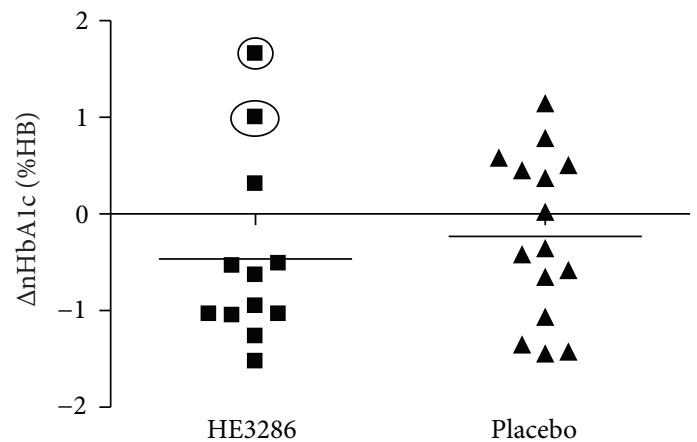

(c)

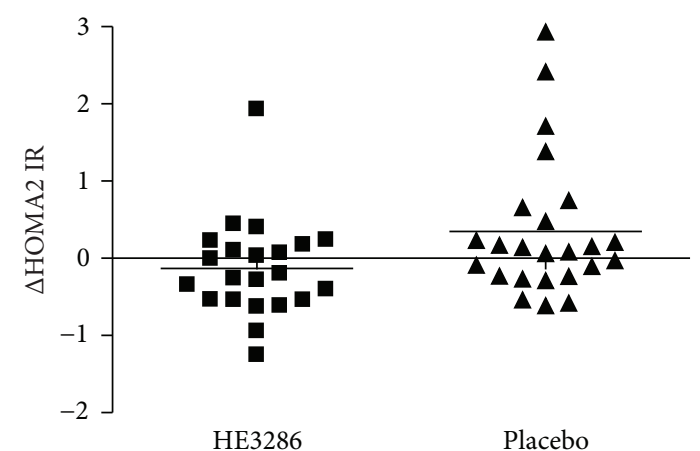

(b)

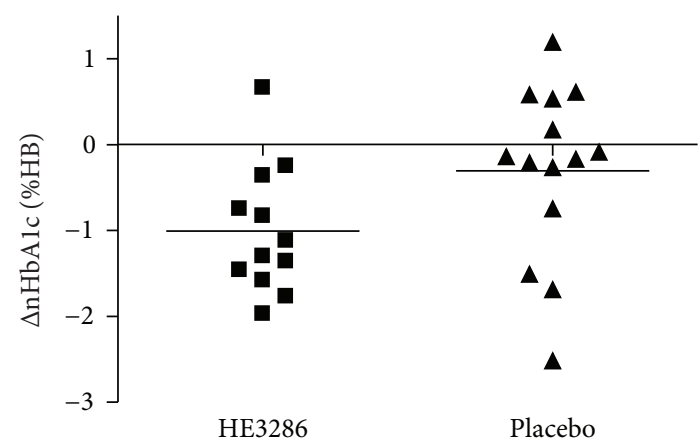

(d)

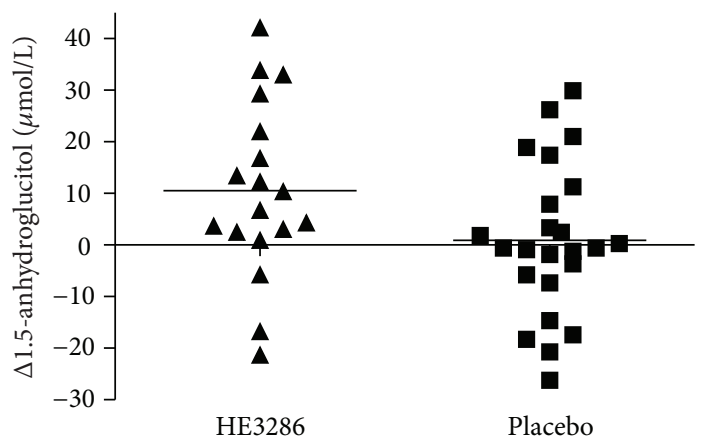

(e)

FIGURE 4: HE3286-0401 distributions of changes by participant. (a) Day 84 cohort 1 baseline $\mathrm{MCP}-1>40$ pmol/L individual subject changes in normalized HbA1c by treatment. (b) Day 84 cohort 1 baseline MCP-1 $>40$ pmol/L individual subject changes in homeostatic model assessment of insulin resistance by treatment. (c) Day 84 cohort 2 baseline BMI $>31 \mathrm{~kg} / \mathrm{m}^{2}$ individual subject changes in normalized HbA1c by treatment. The circles indicate two outliers detected using Mahalanobis distance. (d) Day 112 cohort 2 baseline BMI $>31 \mathrm{~kg} / \mathrm{m}^{2}$ individual subject changes in normalized HbAlc by treatment. (e) Pooled 1,5 anhydroglucitol changes from day 84 cohort 1 and 2 analyses by treatment. Analyses were performed on a subset of patients with available retention samples, predominantly those in cohort 1 with baseline MCP-1 > $40 \mathrm{pmol} / \mathrm{L}$ and in cohort 2 with baseline BMI $>31 \mathrm{~kg} / \mathrm{m}^{2}$.

for cohort 2 placebo subjects as a whole were significantly higher ( $F$ test) for changes in all of the following parameters: day 84 insulin, HOMA2 \%B, and triglycerides, and day 112 insulin and HOMA2 \%B. Variances in placebo were also higher for the BMI $>31 \mathrm{~kg} / \mathrm{m}^{2}$ subgroup for changes in day 84 MCP-1 and triglycerides and day 112 insulin (Table 5).

These differences in distribution and dispersion between groups were not readily evident until day 84 of treatment (data not shown). Together, these findings further support an HE3286 treatment effect that decreases random metabolic effects and restores homeostasis to uncontrolled T2DM patients.

\section{Discussion}

4.1. Study HE3286-0401. This initial clinical trial of HE3286 in diabetes was designed to take all eligible patients with uncomplicated T2DM even though HE3286 was only qualified in animal models of obese diabetes and subsequently only demonstrated activity in obese individuals that present with inflammation-induced insulin resistance. The strategic intent 
TABLE 4: HE3286-0401 treatment effects in obese inflamed subgroups.

\begin{tabular}{|c|c|c|c|c|c|c|}
\hline \multirow{2}{*}{ Group } & \multirow{2}{*}{ Effect } & \multirow{2}{*}{ Value } & \multicolumn{2}{|c|}{ Change } & \multirow{2}{*}{$P$} & \multirow{2}{*}{ Test $^{\mathrm{g}}$} \\
\hline & & & HE3286 & Placebo & & \\
\hline \multirow{10}{*}{$\begin{array}{l}\text { Cohort } 1 \\
\mathrm{MCP}>40^{\mathrm{a}}\end{array}$} & $\Delta \mathrm{HOMA} 2 \mathrm{IR}^{\mathrm{c}}$ & Day 84 mean & -0.1 & +0.4 & 0.02 & \\
\hline & $\Delta \mathrm{C}$-peptide & & -0.03 & +0.1 & 0.04 & \\
\hline & $\Delta \mathrm{Hb}$ & & -0.25 & +0.06 & 0.02 & $t$-test \\
\hline & $\Delta \mathrm{Hct}$ & & -0.06 & +0.09 & 0.02 & \\
\hline & $\triangle \mathrm{RBC}$ & & -0.05 & +0.09 & 0.02 & \\
\hline & $\Delta \mathrm{nHbA1c}$ & Day 84 median & -0.34 & & 0.03 & Wilcoxon \\
\hline & & Day 84 median & & 0.1 & - & \\
\hline & & Day 84 numbers & $17 \Downarrow^{\mathrm{e}} 5 \Uparrow^{\mathrm{f}}$ & $9 \Downarrow 16 \Uparrow$ & 0.0008 & Fisher's Exact \\
\hline & & Day 84 mean & -0.46 & -0.21 & - & \\
\hline & & Day 84 mean-2 outliers ${ }^{\mathrm{d}}$ & -0.82 & -0.21 & 0.04 & $t$-test \\
\hline \multirow{6}{*}{$\begin{array}{l}\text { Cohort } 2 \\
\mathrm{BMI}>31^{\mathrm{b}}\end{array}$} & $\Delta \mathrm{nHbA} 1 \mathrm{c}$ & Day 112 mean & -1.0 & & 0.0007 & \\
\hline & & & & -0.3 & - & $t$-test \\
\hline & & & -1.0 & -0.3 & 0.03 & \\
\hline & & Day 112 median & -1.2 & & 0.002 & Wilcoxon \\
\hline & & & & -0.16 & - & \\
\hline & & & -1.2 & -0.16 & 0.02 & Mann Whitney \\
\hline
\end{tabular}

$\bar{a}$ Participants with baseline monocyte chemoattractant protein greater than the lowest tertile $(40 \mathrm{pmol} / \mathrm{L}) .{ }^{\mathrm{b}}$ Participants with baseline body mass index greater than the median $\left(31 \mathrm{~kg} / \mathrm{m}^{2}\right) .{ }^{\mathrm{c}} \Delta$ : change in; HOMA2 IR: homeostatic assessment model insulin resistance; Hb: hemoglobin; Hct: hematocrit, RBC: red blood cells; nHbAlc: normalized HbA1c (see Section 2 for details); ${ }^{\mathrm{d}}$ Two outliers removed (outliers circled in Figure 4(c), Mahalanobis distance); ${ }^{\mathrm{e}}$ Decrease from zero change; ${ }^{\mathrm{f}}$ Increase from zero change; ${ }^{\mathrm{g}}$ Parametric means and $t$-test used for data with normally distributed data, Nonparametric medians, Wilcoxon, Mann Whitney, and Fisher's Exact test used for abnormally distributed data.

of the study was to identify the responding T2DM population by surveying a broad swath of the constellation of syndromes that are defined by the T2DM condition.

Based on findings in cohort 1 , which indicated low BMI individuals were HE3286 nonresponders, and the inclusion criteria in the second cohort of the trial were modified, concentrating the population to elevated weight (BMI) and inflammatory status (MCP-1). Additional criteria included a requirement for detectable insulin and C-peptide levels. This eliminated the patient population that had progressed to lose significant $\beta$-cell function and who were no longer able to produce insulin, a population clearly not indicated for treatment with an insulin sensitizer. Notably, these criteria were also imposed on clinical trials with the thiazolidinediones (J. Olefsky, personal communication). In addition treatment-naïve patients were recruited in cohort 2 to remove the potential for metformin to blunt the HE3286 treatment effect and consequently amplify the single agent treatment outcome.

We designed this study to test the hypothesis, based on preclinical data and on molecular studies of HE3286 binding partners, that HE3286 would decrease the hyperactivation of NFkB with consequent restoration of insulin signaling $[5,6]$, dependent on its interaction with extracellular signal regulated kinase (ERK) 1 and 2 [7] in addition to other binding partners. ERK1 is an important mediator of inflammation-induced insulin resistance [23-25], insulin receptor substrate (IRS)-1 serine (inhibitory) phosphorylation, and the inhibitory effect of $\mathrm{TNF} \alpha$ on insulin signaling
[26]. HE3286 does not inhibit insulin-mediated ERK activation, but inhibits LPS- and TNF $\alpha$-stimulated ERK hyperactivation, and IRS-1 serine phosphorylation mediated by IKK and JNK [5, 6]. Coincident HE3286-mediated changes in ERK, IKK, JNK, and p38 MAPK signal transduction may explain the preferential responses observed in high adiposity inflamed T2DM patients. Signal transduction pathways in omental fat are altered in obese, compared to lean individuals. In humans, activation of JNK and p38 MAPK was increased in omental fat (compared to paired subcutaneous fat) from obese, but not lean individuals, and this hyperphosphorylation correlated with clinical parameters of hyperglycemia and insulin resistance [27]. It will be important to further clarify the role of ERK in the activity of HE3286.

4.2. HE3286 Correlates. Data analysis presented here demonstrated that the cohort 1 day 84 changes in the primary end point $\mathrm{HbA1c}$ had a significant relationship with expected changes in beta-cell function, fasting glucose, and weight, and also with baseline inflammation status (MCP-1). Surprisingly a relationship with hemoglobin was also detected, a biomarker that is presumed stable over several weeks. Of these covariates associated with HbAlc change, only fasting glucose was significant in placebo patients.

In the enriched cohort 2 population, the HbAlc HE3286 treatment response was no longer dependent on MCP1 but rather BMI with a statistically significant negative 


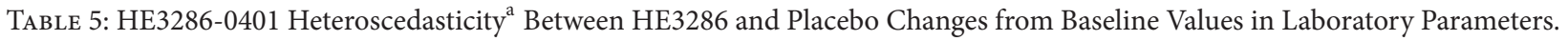

\begin{tabular}{|c|c|c|c|c|c|c|}
\hline \multirow{2}{*}{ Group } & \multirow{2}{*}{ Day } & \multirow{2}{*}{ Parameter } & HE3286 & HE3286 > Placebo & Placebo & Placebo $>$ HE3286 \\
\hline & & & $W$ test $P$ & $F$ test $P$ & $W$ test $P$ & $F$ test $P$ \\
\hline \multirow{6}{*}{ Cohort 1} & \multirow[t]{6}{*}{84} & $\Delta$ Insulin $^{\mathrm{d}}$ & $>0.1$ & $>0.1$ & $<0.0001$ & 0.007 \\
\hline & & $\Delta \mathrm{C}$-peptide & $>0.1$ & $>0.1$ & $<0.0001$ & 0.0495 \\
\hline & & $\Delta$ Fasting glucose & $>0.1$ & $>0.1$ & 0.02 & $>0.1$ \\
\hline & & $\triangle \mathrm{HOMA} 2 \% \mathrm{~B}$ & $>0.1$ & $>0.1$ & $<0.0001$ & $>0.1$ \\
\hline & & $\triangle$ HOMA2 IR & $>0.1$ & $>0.1$ & 0.002 & 0.049 \\
\hline & & $\Delta$ leptin & $>0.1$ & $>0.1$ & 0.005 & $>0.1$ \\
\hline \multirow{3}{*}{$\begin{array}{l}\text { Cohort } 1 \\
\text { MCP-1 }>40^{\mathrm{b}}\end{array}$} & \multirow[t]{3}{*}{84} & $\Delta \mathrm{HbA} 1 \mathrm{c}$ & $>0.1$ & $>0.1$ & 0.006 & $>0.1$ \\
\hline & & $\Delta$ Fasting glucose & $>0.1$ & $>0.1$ & 0.02 & $>0.1$ \\
\hline & & $\triangle \mathrm{HOMA} 2 \% \mathrm{~B}$ & $>0.1$ & $>0.1$ & $<0.0001$ & $>0.1$ \\
\hline \multirow{10}{*}{ Cohort 2} & \multirow[t]{6}{*}{84} & $\Delta \mathrm{nHbA1c}$ & $>0.1$ & $>0.1$ & 0.04 & $>0.1$ \\
\hline & & $\Delta$ Insulin & $>0.1$ & $>0.1$ & $>0.1$ & 0.004 \\
\hline & & $\Delta$ Fasting glucose & $>0.1$ & $>0.1$ & 0.03 & $>0.1$ \\
\hline & & $\triangle \mathrm{HOMA} 2 \% \mathrm{~B}$ & $>0.1$ & $>0.1$ & $>0.1$ & 0.006 \\
\hline & & $\Delta \mathrm{MCP}-1$ & $>0.1$ & $>0.1$ & 0.005 & $>0.1$ \\
\hline & & $\Delta$ Triglycerides & $>0.1$ & $>0.1$ & $<0.0001$ & 0.007 \\
\hline & \multirow[t]{4}{*}{112} & $\Delta \mathrm{nHbA1c}$ & $>0.1$ & $>0.1$ & 0.0007 & $>0.1$ \\
\hline & & $\Delta$ Insulin & $>0.1$ & $>0.1$ & $>0.1$ & 0.008 \\
\hline & & $\Delta$ Fructosamine & $>0.1$ & $>0.1$ & 0.002 & $>0.1$ \\
\hline & & $\triangle \mathrm{HOMA} 2 \% \mathrm{~B}$ & $>0.1$ & $>0.1$ & $<0.0001$ & 0.01 \\
\hline \multirow{8}{*}{$\begin{array}{l}\text { Cohort } 2 \\
\text { BMI }>31^{c}\end{array}$} & \multirow[t]{3}{*}{84} & $\triangle \mathrm{HOMA} 2 \% \mathrm{~B}$ & $>0.1$ & $>0.1$ & 0.007 & $>0.1$ \\
\hline & & $\Delta \mathrm{MCP}-1$ & $>0.1$ & $>0.1$ & $>0.1$ & 0.009 \\
\hline & & $\Delta$ Triglycerides & $>0.1$ & $>0.1$ & $>0.1$ & 0.001 \\
\hline & \multirow[t]{5}{*}{112} & $\Delta$ Insulin & $>0.1$ & $>0.1$ & $<0.0001$ & 0.001 \\
\hline & & $\Delta \mathrm{C}$-peptide & $>0.1$ & $>0.1$ & $<0.0001$ & $>0.1$ \\
\hline & & $\triangle \mathrm{HOMA} 2 \% \mathrm{~B}$ & $>0.1$ & $>0.1$ & $<0.0001$ & $>0.1$ \\
\hline & & $\triangle$ HOMA2 IR & $>0.1$ & $>0.1$ & $<0.0001$ & $>0.1$ \\
\hline & & & $>0.1$ & $>0.1$ & $>0.1$ & $>0.1$ \\
\hline
\end{tabular}

${ }^{\mathrm{a}}$ Heteroscedasticity describes differences in variances between groups. ${ }^{\mathrm{b}}$ Participants with baseline monocyte chemoattractant protein greater than the lowest tertile $\left(40 \mathrm{pmol} / \mathrm{L}\right.$, see results). ${ }^{\mathrm{c}}$ Participants with baseline body mass index greater than the median $\left(31 \mathrm{~kg} / \mathrm{m}^{2}\right.$, see results). ${ }^{\mathrm{d}}$ Abbreviations: $\Delta:$ change in; HOMA2 \%B: homeostatic model assessment of pancreatic beta cell function; HOMA2 IR: homeostatic model assessment of insulin resistance; HbA1c: hemoglobin A1c; nHbA1c: HbAlc normalized to 84 day average hemoglobin mass; MCP-1: monocyte chemoattractant protein-1.

correlation; the higher the BMI the larger the effect on HbAlc decline. Higher BMI subjects presented with higher MCP1. The cohort 2 outcome remained correlated with expected changes in $\beta$-cell function and with fasting glucose.

Thus the general population enrolled in cohort 1 was a very different ensemble of participants than those enrolled in cohort 2. While the relationships of change in HbA1c with changes in $\beta$-cell function and fasting glucose remained, the relationship to weight loss was not seen in the cohort 2 participants selected with higher BMI inclusion criteria.

4.3. Placebo Correlates. Cohort 1 placebo group HbA1c change was dependent only on baseline inflammation status (TNF $\alpha$ and day $84 \mathrm{TNF} \alpha$ change). In cohort 2, there were no placebo correlates to $\mathrm{HbA1c}$ change. Importantly, fasting glucose change was not correlated with HbA1c change in this group, indicating that glucose levels were uncoupled from the HbAlc surrogate marker. Rather, placebo HbAlc variance $(\mathrm{CV})$ was correlated strictly with inflammation in both cohorts. This was evidenced by correlation to baseline TNF $\alpha$ in cohort 1 and dependent on both changes in MCP1 and surprisingly hemoglobin $\mathrm{CV}$ in cohort 2 . $\mathrm{Hb} \mathrm{CV}$ was in turn dependent on baseline MCP-1 (TNF $\alpha$ was not measured).

In cohort 1 this later dependency on $\mathrm{Hb} \mathrm{CV}$ was not detected perhaps due to the heterogeneity of the more general patient population (including nonobese and noninflamed diabetics). In cohort 2, placebo $\mathrm{HbA1c} \mathrm{CV}$ was negatively correlated with weight change, indicating that higher weight led to increased variance. Cohort 2 placebo weight loss was unexpectedly unrelated to $\mathrm{HbA1c}$ and glucose control. Since placebo $\mathrm{HbA} 1 \mathrm{c} \mathrm{CV}$ was correlated with $\mathrm{TNF} \alpha$ change in cohort 1, the weight loss associated with higher $\mathrm{HbA1c} C \mathrm{~V}$ in the cohort 2 placebo group is presumed to be related 
to inflammation effects on satiety or metabolism leading to changes in caloric intake and energy balance.

4.4. HE3286 Treatment Effects. The significant correlation of changes in $\mathrm{HbA} 1 \mathrm{c}$ and hemoglobin was an unexpected observation as hemoglobin is considered an invariant biomass from which HbAlc is formed as a reflection of total hyperglycemia and therefore its status as an FDA approved biomarker. Inspection of individual patient $\mathrm{HbAlc}$ changes revealed a high degree of intravisit variance, contrary to its presumed highly controlled and stable total body mass. Further exploration of the hematopoietic elements gathered with the safety data demonstrated these variant effects were not only on the hemoglobin mass but also on other components such as RBC, hematocrit, mean red cell volume, mean corpuscular hemoglobin and platelets, as well as a variety of metabolic parameters including glucose and cholesterol.

Unexpected variance in metabolic and hematologic parameters related to the effects of chronic low-grade inflammation in uncontrolled obese diabetes produced a significant barrier to these analyses and data interpretation. The variances that caused differing distributions and dispersions between treatment and placebo groups' coupled with the HE3286 treatment effect presented significant statistical challenges. Statistically random effects in the treatment-naïve placebo group were demonstrated for day 84 changes in glucose and in the key surrogate parameters hemoglobin, $\mathrm{HbA} 1 \mathrm{c}$ and HOMA2 \% B. The increasing variances in individual $\mathrm{HbAlc}$ change with metabolic disease progression demonstrated median changes of zero for dyslipidemic, IGT, and T2DM patients. Statistically, random effects are assumed to be the realization of a normal distribution with a mean of zero and a variance that can be estimated. In order to investigate HE3286 treatment effects, we were prompted to remove this random component by normalizing $\mathrm{HbAlc}$ to the day 84 average total hemoglobin mass (mean 84-day change of zero) for each patient.

In the broadly defined population of metformin-treated T2DM patients (cohort 1), the HE3286 responsive patient population was found in the upper two tertiles of the inflammation marker MCP-1 ( $\geq 40 \mathrm{pmol} / \mathrm{L})$. In the inflamed treatment-naïve patients' population studied in cohort 2 , the responding population was found above the median BMI (obese subjects, $>31 \mathrm{~kg} / \mathrm{m}^{2}$ ). The magnitude of the treatment response was indeed greater in the treatment-naive (cohort 2) than metformin-treated patients.

In both cohort 1 and 2, HE3286 treatment was associated with a total $\mathrm{Hb}$ mass normalization evidenced by day 84 data distributions and decreased variances in numerous metabolic and erythroid values. For several of these dysregulated parameters, HE3286 did not show a significant correction until day 84 . We interpret these results to indicate that HE3286, via its anti-inflammatory activity, decreased inflammation-driven metabolic dysregulation.

HE3286 showed a significant effect to improve insulin resistance in IGT subjects [9] and to decrease HOMA2 IR in cohort 1 T2DM patients, but not in cohort 2. It is possible that since cohort 2 was naïve, previously untreated T2DM patients and showed higher variances, additional improvements would be observed with longer treatment time frames or drug combination therapy.

4.5. Metabolic Disease, Variance, and Random Effects. The relationship between inflammation and increased variances in erythroid and metabolic laboratory parameters was investigated in clinical settings of increasing chronic low-grade inflammation, adiposity, and metabolic dysregulation. Compared to a healthy group, significantly increased variances were observed for hematocrit, and $\mathrm{HbAlc}$ for dyslipidemic, IGT, and T2DM patients. RBC and hemoglobin values were also significantly variable, and the fasting glucose was variable in both dyslipidemic and T2DM patients. In treatment-naive T2DM, high variances and random effects were observed in a large number of metabolic and hematologic parameters that the medical community relies on for medical diagnoses. These changes were correlated with increased inflammatory mediators. This data supports our hypothesis that, in obese subjects, adipose tissue inflammation contributes to both metabolic and hematologic dysregulation within the same individuals.

This is the first clinical report of extreme fluctuations in the marker $\mathrm{HbAlc}$ in patients with uncontrolled type 2 diabetes mellitus, but there are published data for type 1 diabetes mellitus (T1DM). Fluctuations in \%HbAlc of more than $1 \%$ occurred in $50 \%$ of the patients year to year, and over 9 years the minimum-maximum range was $>3 \%$ and $>5 \% \mathrm{HbAlc}$ in $55 \%$ and $11 \%$ of patients, respectively, [17]. In T1DM subjects followed for 4 years, there was high CV for intraindividual $\mathrm{HbAlc}$ measurements $(15.5 \pm 8.1 \%)$, which was lower for patients with good glycemic control. Intrasubject variations of fasting glucose and HbAlc (HbAlc 6-8\%, with $<10 \%$ variation in $\mathrm{HbAlc}$ over the last two months) were determined in healthy subjects and T1DM patients with good glycemic control [18]. Glucose intrasubject CV, were $5.4 \%$ (range 4.6-6.0) for healthy and 30.5\% (26.7-35.5) for T1DM. HbA1c CV, were $1.2 \%(1.1-1.4)$ for healthy and 1.7\% (1.5-1.9) for T1DM with good glycemic control. Longitudinal changes in T1DM glycemic control gave a significant positive association between baseline HbAlc and $\mathrm{CV}$ for intraindividual HbAlc $(P<0.01)$ [19].

HbAlc is a useful marker for detection of patients with elevated fasting and postprandial glucose. The ADA recommends that anyone with $\mathrm{HbAlc}>7$ be treated with additional agents to return them to a glucose-controlled state. In this specific patient population of type 2 diabetes with $\mathrm{HbAlc}$ that is uncontrolled according to the ADA recommendations, the authors have found that the basic hypothesis of stable hemoglobin and red cell lifespan allowing extrapolation from $\mathrm{HbAlc}$ change and glucose control is flawed, that intravisit fluctuations can be large, and that a change in HbAlc values between two visits is unlikely to reflect a meaningful therapeutic effect on glucose control in this uncontrolled population. Thus, in clinical efficacy studies in patients with poorly controlled $\mathrm{HbAlc}$, the authors recommend that additional tests of glucose control be used for determination of efficacy of new antidiabetic therapies. Numerous publications argue for the improved management by using continuous glucose monitoring, and for the Ptime-averaged effects of 
using 1,5-anhydroglucitol [28] to better understand variation in glucose control.

4.6. HE3286-0401 Conclusions. The hypotheses tested in this study appear to be borne out in the high adiposity T2DM patient. HE3286 preferentially improved clinical parameters in obese inflamed insulin-resistant T2DM patients. Since inflammatory changes were driving HbAlc changes in the placebo group for both cohorts 1 and 2, the changes observed with HE3286 treatment appear to be due to its antiinflammatory activity (i.e., to break the cycle of inflammatory kinase-mediated inhibition of insulin receptor signaling). Furthermore, T2DM subjects that lack chronic, low-grade inflammation lack the specific lesion in the insulin receptor signaling pathway that HE3286 was developed to interdict. Their glucose intolerance arises for other reasons, and therefore they are unaffected by HE3286.

Obese type 2 diabetic incidence is increasing at an alarming rate. Regaining glucose control and metabolic regulation and preventing or delaying macrovascular and microvascular complications could help to contain rising health care costs for end-stage diabetes complications. Understanding which patients are to benefit from a new therapy is now a regulatory consideration. The FDA has published the Critical Path Initiative, with personalized medicine, or the patient-specific information to individualize therapy and disease management as a major theme, and published on the importance of clinical validation of personalized medicine selection criteria in diabetes [29]. Based on estimates of obese, inflamed diabetics in the future at approximately 50\% (J. Olefsky, personal communication), HE3286 offers a potentially important personalized medicine for these subjects.

HE3286 is active at low (hormonal level) doses and is an anti-inflammatory insulin sensitizer with a toxicology profile conducive for chronic daily use [1]. In the responsive subpopulations HE3286 significantly decreased HbA1c compared to placebo, by day 84 in metformin-treated subjects with high MCP-1, and by day 112 in treatment-naïve subjects with high BMI. The data presented here in uncontrolled T2DM patients make a compelling argument for further testing of HE3286 in the high adiposity, inflamed T2DM patient subset, using oral glucose tolerance testing, 1,5-AH, or continuous glucose monitoring to assess treatment effects. The correlation or lack thereof with the surrogate marker HbAlc should be confirmed in these uncontrolled patients.

\section{Conflict of Interests}

C. Reading, J. F. Riveros, D. Stickney and J. Frincke are or were employees of Harbor Therapeutics, Inc. J. Frincke is a member of the board of directors. C. Reading and J. Frincke are shareholders. The authors have no other conflicts of interest.

\section{References}

[1] C. N. Ahlem, M. R. Kennedy, T. M. Page et al., "Studies of the pharmacology of $17 \alpha$-ethynyl-androst-5-ene- $3 \beta, 7 \beta, 17 \beta$-triol, a synthetic anti-inflammatory androstene," International Journal of Clinical and Experimental Medicine, vol. 4, no. 2, pp. 119-135, 2011.

[2] K. E. Wellen and G. S. Hotamisligil, "Obesity-induced inflammatory changes in adipose tissue," Journal of Clinical Investigation, vol. 112, no. 12, pp. 1785-1788, 2003.

[3] J. M. Olefsky and C. K. Glass, "Macrophages, inflammation, and insulin resistance," Annual Review of Physiology, vol. 72, pp. 219-246, 2009.

[4] S. E. Shoelson, J. Lee, and A. B. Goldfine, "Inflammation and insulin resistance," Journal of Clinical Investigation, vol. 116, no. 7, pp. 1793-1801, 2006.

[5] M. Lu, D. Patsouris, P. Li et al., "A new antidiabetic compound attenuates inflammation and insulin resistance in Zucker diabetic fatty rats," American Journal of Physiology, vol. 298, no. 5, pp. E1036-E1048, 2010.

[6] T. Wang, S. Villegas, Y. Huang et al., "Amelioration of glucose intolerance by the synthetic androstene HE3286: link to inflammatory pathways," Journal of Pharmacology and Experimental Therapeutics, vol. 333, no. 1, pp. 70-80, 2010.

[7] C. L. Reading, J. M. Frincke, and S. K. White, "Molecular targets for 17alpha-ethynyl-5-androstene-3beta, 7beta, 17betatriol, an anti-inflammatory agent derived from the human metabolome," PLoS One, vol. 7, Article ID e32147, 2012.

[8] N. Barbarroja, R. López-Pedrera, M. D. Mayas et al., “The obese healthy paradox: is inflammation the answer?" Biochemical Journal, vol. 430, no. 1, pp. 141-149, 2010.

[9] C. Reading, D. Stickney, J. Flores-Riveros et al., "A synthetic anti-inflammatory sterol improves insulin sensitivity in insulinresistant obese impaired glucose tolerance subjects," Obesity. In press.

[10] R. M. Cohen, R. S. Franco, P. K. Khera et al., "Red cell life span heterogeneity in hematologically normal people is sufficient to alter HbA1c," Blood, vol. 112, no. 10, pp. 4284-4291, 2008.

[11] M. Ghashghaeinia, J. C. Cluitmans, A. Akel et al., "The impact of erythrocyte age on eryptosis," British Journal of Haematology, vol. 157, pp. 606-614, 2010.

[12] B. Bistrian, "Systemic response to inflammation," Nutrition Reviews, vol. 65, no. 12, pp. S170-S172, 2007.

[13] N. Grossin, M. P. Wautier, and J. L. Wautier, "Red blood cell adhesion in diabetes mellitus is mediated by advanced glycation end product receptor and is modulated by nitric oxide," Biorheology, vol. 46, no. 1, pp. 63-72, 2009.

[14] H. Vlassara, J. Valinsky, and M. Brownlee, "Advanced glycosylation endproducts on erythrocyte cell surface induce receptormediated phagocytosis by macrophages. A model for turnover of aging cells," Journal of Experimental Medicine, vol. 166, no. 2, pp. 539-549, 1987.

[15] K. P. Conroy, I. M. Davidson, and M. Warnock, "Pathogenic obesity and nutraceuticals," Proceedings of the Nutrition Society, vol. 70, pp. 426-438, 2011.

[16] F. León-Velarde, F. C. Villafuerte, and J. P. Richalet, "Chronic mountain sickness and the heart," Progress in Cardiovascular Diseases, vol. 52, no. 6, pp. 540-549, 2010.

[17] B. M. Singh, C. Mcnamara, and P. H. Wise, "High variability of glycated hemoglobin concentrations in patients with IDDM followed over 9 years: what is the best index of long-term glycemic control?” Diabetes Care, vol. 20, no. 3, pp. 306-308, 1997.

[18] S. Carlsen, P. H. Petersen, S. Skeie, O. Skadberg, and S. Sandberg, "Within-subject biological variation of glucose and 
$\mathrm{HbA}(1 \mathrm{c})$ in healthy persons and in type 1 diabetes patients," Clinical Chemistry and Laboratory Medicine, vol. 49, pp. 1501-1507, 2011.

[19] R. Jorde and J. Sundsfjord, "Intra-individual variability and longitudinal changes in glycaemic control in patients with type 1 diabetes mellitus," Diabetic Medicine, vol. 17, no. 6, pp. 451-456, 2000.

[20] D. R. Stickney, J. R. Groothuis, C. Ahlem et al., "Preliminary clinical findings on NEUMUNE as a potential treatment for acute radiation syndrome," Journal of Radiological Protection, vol. 30, no. 4, pp. 687-698, 2010.

[21] D. R. Stickney, C. N. Ahlem, E. Morgan, C. L. Reading, N. Onizuka, and J. M. Frincke, "Phase I and Phase II clinical trials of androst-5-ene-3 $\beta, 7 \beta, 17 \beta$-triol," American Journal of Translational Research, vol. 3, no. 3, pp. 275-283, 2011.

[22] J. Sun, J. T. Dou, X. L. Wang et al., "Correlation between 1, 5 -anhydroglucitol and glycemic excursions in type 2 diabetic patients," Chinese Medical Journal, vol. 124, pp. 3641-3645, 2011.

[23] J. Jager, V. Corcelle, T. Grémeaux et al., "Deficiency in the extracellular signal-regulated kinase 1 (ERK1) protects leptindeficient mice from insulin resistance without affecting obesity," Diabetologia, vol. 54, no. 1, pp. 180-189, 2011.

[24] S. J. Lee, P. T. Pfluger, J. Y. Kim et al., "A functional role for the p62-ERK1 axis in the control of energy homeostasis and adipogenesis," EMBO Reports, vol. 11, no. 3, pp. 226-232, 2010.

[25] A. Rodriguez, A. Durán, M. Selloum et al., "Mature-onset obesity and insulin resistance in mice deficient in the signaling adapter p62," Cell Metabolism, vol. 3, no. 3, pp. 211-222, 2006.

[26] B. Emanuelli, D. Eberlé, R. Suzuki, and C. R. Kahn, "Overexpression of the dual-specificity phosphatase MKP-4/DUSP-9 protects against stress-induced insulin resistance," Proceedings of the National Academy of Sciences of the United States of America, vol. 105, no. 9, pp. 3545-3550, 2008.

[27] N. Bashan, K. Dorfman, T. Tarnovscki et al., "Mitogen-activated protein kinases, inhibitory- $\kappa \mathrm{B}$ kinase, and insulin signaling in human omental versus subcutaneous adipose tissue in obesity," Endocrinology, vol. 148, no. 6, pp. 2955-2962, 2007.

[28] C. M. Hightower, B. Y. Salazar Vazquez, S. Woo Park et al., "Integration of cardiovascular regulation by the blood/endothelium cell-free layer," Wiley Interdisciplinary Reviews: Systems Biology and Medicine, vol. 3, pp. 458-470, 2011.

[29] C. C. Harper, "Personalized medicine in diabetes: regulatory considerations," Journal of Diabetes Science and Technology, vol. 3, no. 4, pp. 739-742, 2009 


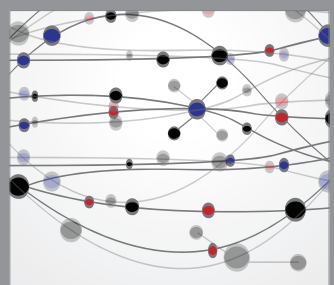

The Scientific World Journal
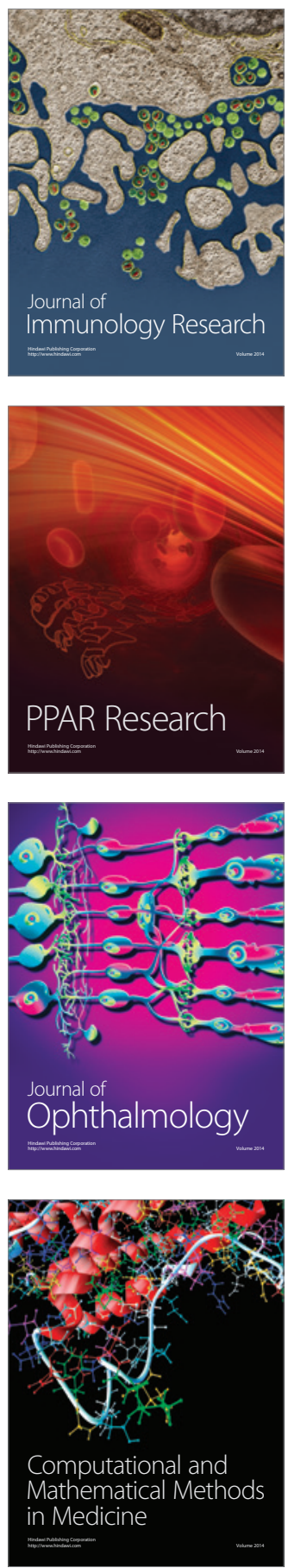

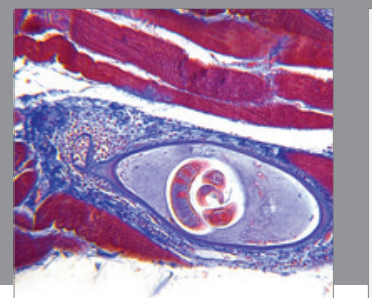

Gastroenterology

Research and Practice
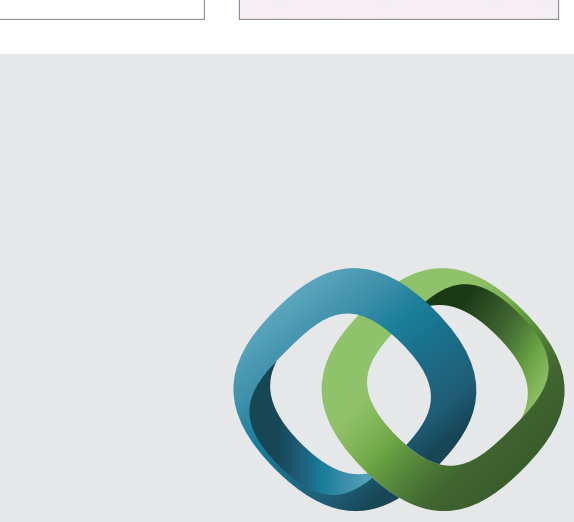

\section{Hindawi}

Submit your manuscripts at

http://www.hindawi.com
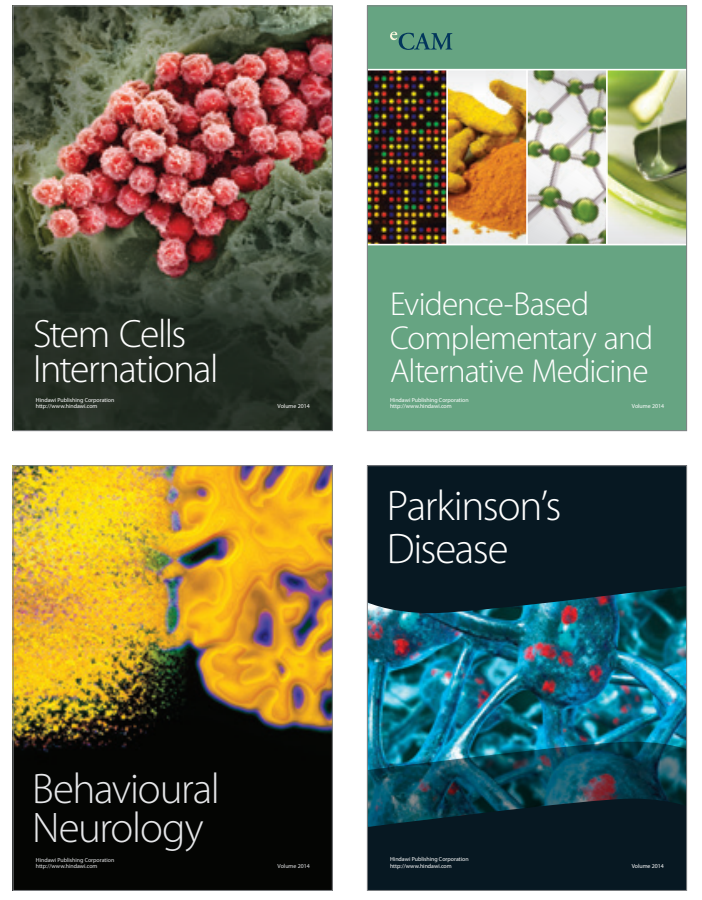
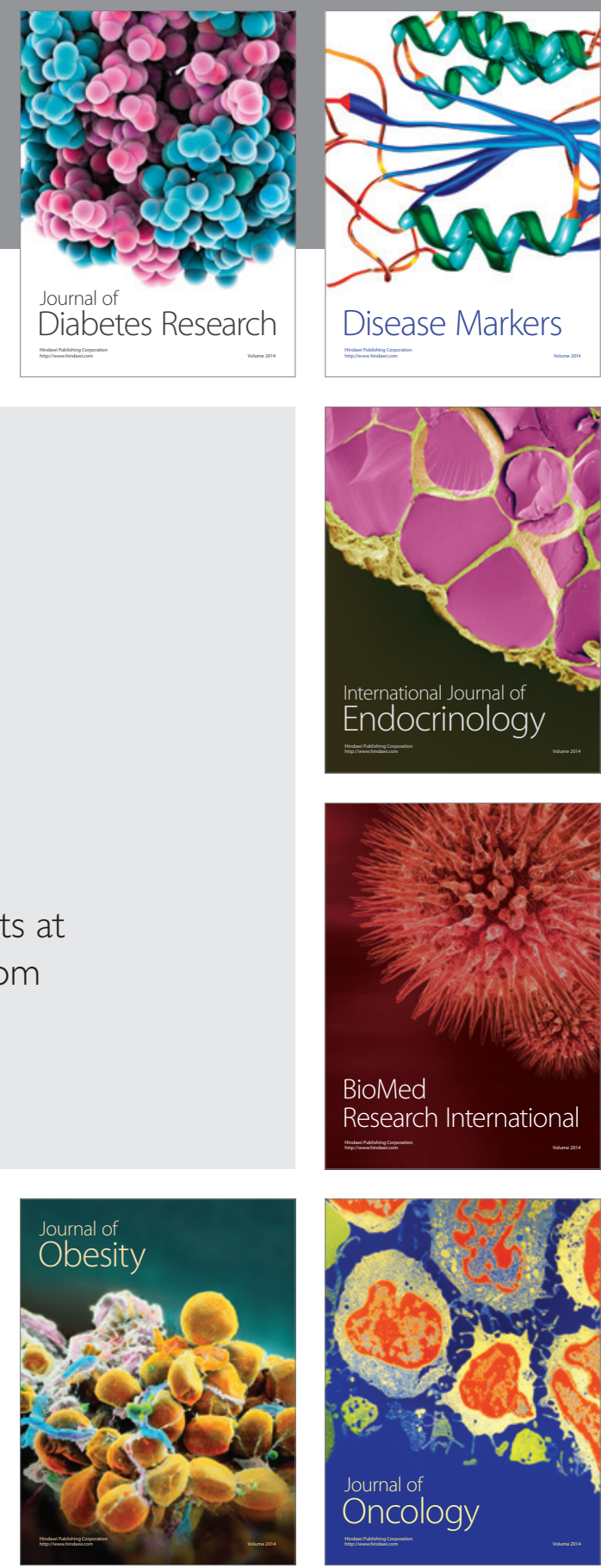

Disease Markers
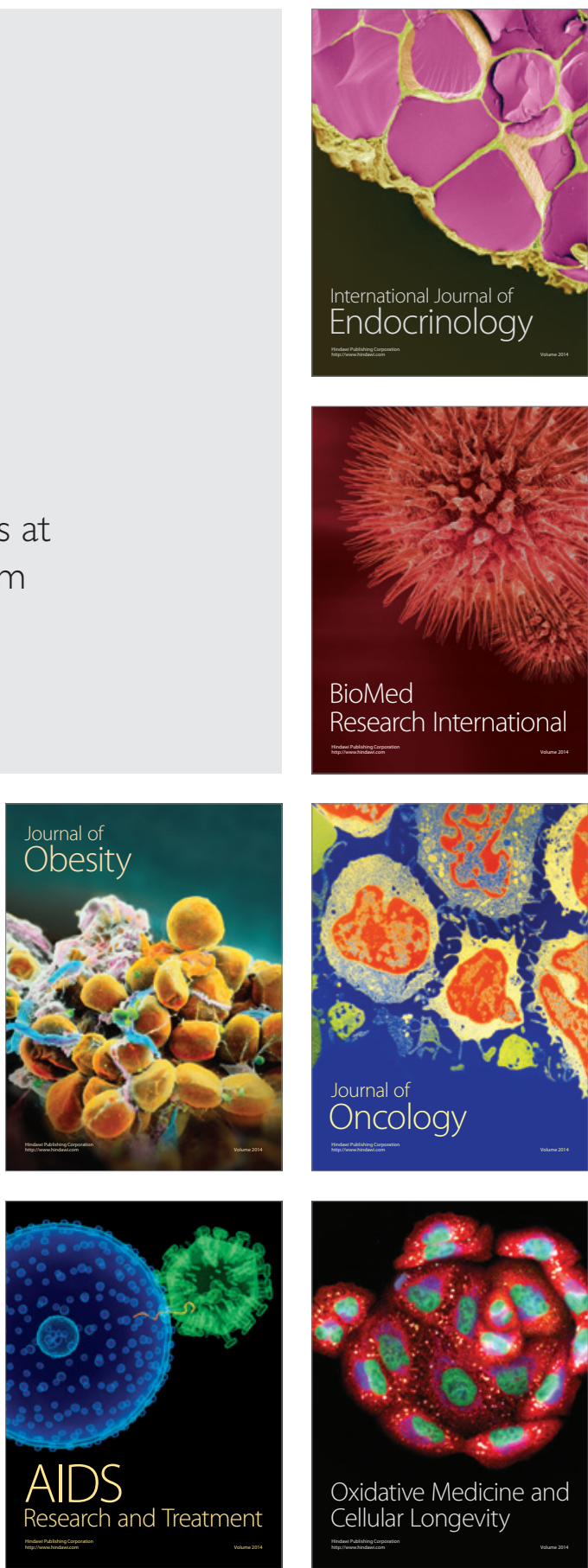